\title{
CARACTERIZACIÓN TAXONÓMICA Y PALEOECOLÓGICA DE LA INGRESIÓN DEL HOLOCENO EN EL CANAL BEAGLE (TIERRA DEL FUEGO), EN BASE A LAS ASOCIACIONES DE DINOQUISTES Y OTROS PALINOMORFOS ACUÁTICOS
}

\author{
MARÍA SOLEDAD CANDEL \& ANA MARÍA BORROMEI \\ INGEOSUR-CONICET, Departamento de Geología, Universidad Nacional del Sur, San Juan, 670, B8000ICN, \\ BahíaBlanca, Argentina.scandel@uns.edu.ar,borromei@criba.edu.ar
}

\begin{abstract}
TAXONOMIC AND PALEOECOLOGICAL CHARACTERIZATION OF HOLOCENE INGRESSION IN THE BEAGLE CHANNEL (TIERRA DEL FUEGO), BASED ON DINOCYST ASSEMBLAGES AND OTHER AQUATIC PALYNOMORPHS. On the southern coast of Tierra del Fuego, Argentina, the Holocene marine ingression took place around $8,000 \mathrm{C}^{14}$ years $\mathrm{BP}$, and it is represented by several discontinuous marine terraces along the north coast of the Beagle Channel. During the beginning of the marine incursion, the aquatic assemblages developed in Bahía Lapataia and Río Varela localities, were characterized by a scarce participation of marine components, especially of dinoflagellate cysts. After $6,000 \mathrm{C}^{14}$ years BP, in Río Ovando and Albufera Lanushuaia localities, an increase in the number of aquatic species was observed with a major participation of Peridiniales dinocysts in the associations, suggesting the establishment of marginal marine environments with low to moderate salinities and high nutrient content in the water. The analysis of the dinoflagellate cysts and other aquatic palynomorphs assemblages recorded in the marine sediments from northern coast of the Beagle Channel, indicate that the environmental conditions during the middle-late Holocene correspond to fjord (estuarine) environments close to ice fields affected by glacier meltwater discharge with anomalously low salinities. These aquatic assemblages identified have a similar composition to those observed in modern sediments of the Beagle Channel.
\end{abstract}

Key words: aquatic palynomorphs, Holocene marine sediments, Beagle Channel.

RESUMO - Na costa sul da Tierra del Fuego, Argentina, a ingressão marinha holocena (datada em torno de 8.000 anos AP pelo método $\mathrm{C}^{14}$ ) é representada por vários terraços marinhos descontínuos ao longo da costa norte do Canal de Beagle. No início da ingressão marinha, nas localidades de Baía Lapataia e Rio Varela, as associações aquáticas caracterizavam-se por uma escassa participação de elementos marinhos, em especial de cistos de dinoflagelados. A partir de 6.000 anos AP, nas localidades de Albufera Lanushuaia e Rio Ovando, foi observado um aumento no número de palinomorfos aquáticos, com maior participação das associações de dinocistos da Ordem Peridiniales, sugerindo o estabelecimento de ambientes marinhos marginais com salinidade baixa a moderada e alto conteúdo de nutrientes. A análise das associações de dinocistos e de outros palinomorfos aquáticos, registrados na costa norte do Canal de Beagle, indica que as condições ambientais estabelecidas durante o Holoceno médio-tardio correspondem a ambientes de fiordes (estuarinos), próximos a glaciares influenciados pela descarga de águas de degelo, com salinidades anomalamente baixas. As associações aquáticas identificadas nos sedimentos holocênicos apresentam uma composição similar àquelas observadas nos sedimentos atuais do Canal de Beagle.

Palavras-chave: palinomorfos aquáticos, sedimentos marinhos holocênicos, Canal de Beagle.

\section{INTRODUCCIÓN}

El estudio del microplancton de pared orgánica, principalmente quistes de dinoflagelados, tiene especial interés para efectuar evaluaciones paleoambientales e inferencias paleoclimáticas. Numerosos estudios han demostrado la existencia de una estrecha relación entre las asociaciones de quistes de dinoflagelados (o dinoquistes) y las condiciones del agua de mar como, temperatura, salinidad, disponibilidad de nutrientes, productividad primaria y profundidad de las masas de agua, estacionalidad y extensión de la cubierta de hielo marino (de Vernal et al., 2001, 2005; Radi \& de Vernal, 2008).
Por lo tanto, las variaciones en su registro son interpretadas en función de cambios oceanográficos y atmosféricos.

En el Hemisferio Sur, aún son escasos los estudios sobre las asociaciones de dinoquistes y otros palinomorfos acuáticos en depósitos cuaternarios (McMinn, 1992, 1995; McMinn \& Wells, 1997; Marret \& de Vernal, 1997; Harland \& Pudsey, 1999; Harland et al., 1998; Marret et al., 2001). Para la costa atlántica de Argentina y plataforma continental pueden mencionarse los estudios realizados por Grill \& Guerstein (1995), Grill \& Quattrocchio (1996), Gómez et al. (2005), Borel \& Gómez (2006), Borel et al. (2006), Borel (2007) y Vilanova et al. (2008). En particular, para la Isla 
Grande de Tierra de Fuego, los estudios sobre microplancton marino de pared orgánica han sido llevados a cabo en el área del Canal Beagle sobre sedimentos de fondo de canal (Candel, 2010; Candel et al., 2012) y sedimentos marinos holocenos aflorantes sobre la costa norte del canal (Borromei \& Quattrocchio, 2001, 2007; Grill et al., 2002; Candel, 2010; Candel et al., 2009, 2011, 2013; Rabassa et al., 2009).

El objetivo del presente trabajo es caracterizar el ambiente de depositación existente durante la ingresión marina del Holoceno en el Canal Beagle, Tierra del Fuego. Con tal fin, se presentan las asociaciones del microplancton de pared orgánica (quistes de dinoflagelados, acritarcos y otras algas) y sus implicancias paleoecológicas que han sido identificadas en los depósitos marinos fósiles provenientes de localidades ubicadas sobre la costa norte de dicho canal.

\section{ÁREA DE ESTUDIO}


Figura 1) se encuentra ubicado al sur de la Isla Grande de Tierra del Fuego y conecta el extremo sur de los océanos Pacífico y Atlántico. La costa norte corresponde a la Isla Grande de Tierra del Fuego, mientras que la costa sur comprende las islas Hoste y Navarino, a su vez separadas por el Canal Murray. El canal es un antiguo valle tectónico que fue ocupado completamente por el hielo durante el Último Máximo Glacial (ca. 25 ka AP, Rabassa et al., 2011). El Glaciar Beagle provenía del manto de hielo de montaña de la Cordillera Darwin, recibiendo glaciares tributarios desde los circos y valles interiores de los cordones montañosos de ambas márgenes y se extendía hacia el sector este del Canal Beagle, hasta alcanzar su posición más externa al este de la localidad Punta Moat (Rabassa et al., 1990). En períodos postglaciales, con el retroceso de los hielos, las áreas deprimidas fueron ocupadas por lagos proglaciarios hasta que se produjo el ascenso relativo del nivel del mar, originando una red de fiordos y canales marinos acompañados por el desarrollo, en algunos sectores, de playas marinas. Si bien aún es incierta el área por donde el agua de mar ingresó dentro de este valle glacial, se postula que podría haber sido a través del Canal Murray el cual se encuentra ubicado inmediatamente al sur de Bahía Lapataia (Figura 1), encontrándose en esta zona las edades radiocarbónicas más antiguas de los depósitos marinos del Canal Beagle (Rabassa et al., 2009). El pasaje de lago proglacial a fiordo habría tenido lugar hacia los 8.200 años $C^{14}$ $\mathrm{AP}$, quedando el ambiente marino completamente establecido al menos a los 7.900 años $C^{14}$ AP (Rabassa et al., 1986).

Esta incursión marina está representada por depósitos marinos discontinuos a lo largo de la costa norte del Canal Beagle. Dadas las características topográficas de la región estos depósitos no se desarrollan en su ancho y no superan en

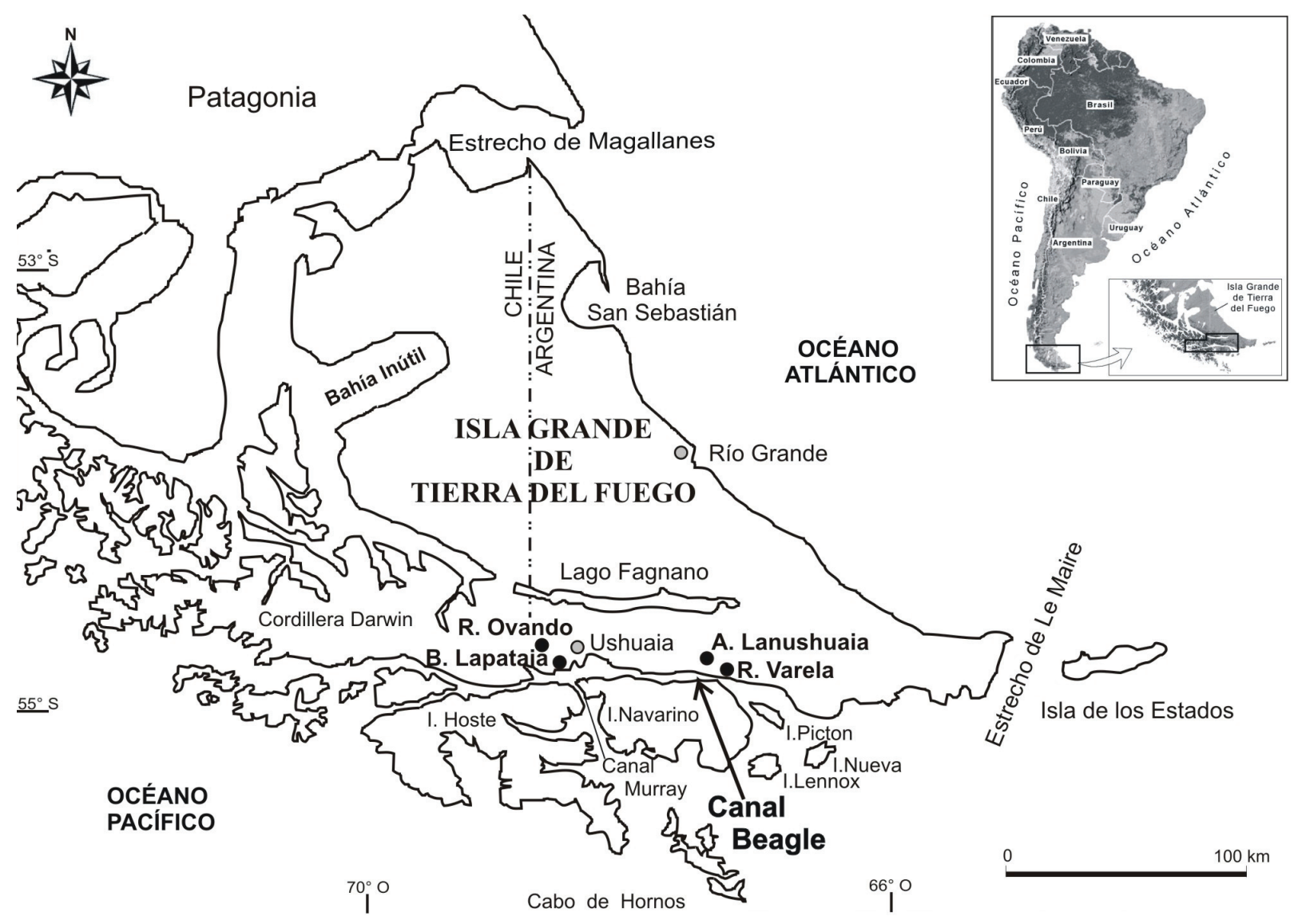

Figura 1. Mapa de ubicación del área de estudio.

Figure 1. Location map of the study area. 
general escasos metros, excepto en el sector occidental, donde la presencia del Lago Roca y el Archipiélago Cormoranes habrían permitido la entrada del mar al menos $4 \mathrm{~km}$ tierra adentro (Gordillo, 1993). Entre Bahía Lapataia e Isla Gable se han reconocido tres niveles de playas holocenas ubicados a 8-10, 4-6 y 1,5-3 m s.n.m., respectivamente (Gordillo et al., 1992; Gordillo, 1993; Rabassa et al., 2000; 2009). Mientras que hacia el E del canal, en la zona de Río Varela, los depósitos marinos holocenos se encuentran sumergidos bajo la línea de mareas o sepultados por secuencias fluviales en las cercanías a las desembocaduras de los ríos (Grill et al., 2002; Rabassa et al., 2004). El control depositacional de este sistema marino es atribuido en parte a factores glacioeustáticos y en parte a movimientos neotectónicos (Rabassa et al., 1992; Bujalesky et al., 2004). Probablemente, las fluctuaciones glacioeustáticas y los movimientos corticales glacioisostáticos habrían actuado hasta los 5.000 años AP, momento en el cual el nivel eustático del mar se estabiliza, y la actividad neotectónica sería posterior a los 5.000 años AP continuando hasta la actualidad (Gordillo, 1993; Rabassa et al., 2009).

\section{MATERIAL Y MÉTODOS}

El material analizado procede de cuatro secciones marinas fósiles aflorantes sobre la costa norte del Canal Beagle, Tierra del Fuego. Ellas son: en el sector O del canal (Figura

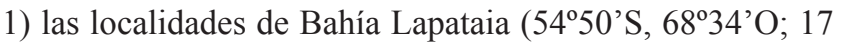
muestras estudiadas) y Río Ovando (54ํำ $51^{\prime} \mathrm{S}, 68^{\circ} 35^{\circ} \mathrm{O} ; 21$ muestras estudiadas), y en el sector E del Canal Beagle (Figura 1) las localidades de Albufera Lanushuaia ( $54^{\circ} 52^{\prime} 04,4^{\prime \prime} \mathrm{S}$, $67^{\circ} 60^{\prime} 44,99^{\prime \prime} \mathrm{O} ; 27$ muestras estudiadas) y Río Varela (5452'S, $67^{\circ} 11^{\prime} \mathrm{O} ; 18$ muestras estudiadas). La información cualicuantitativa correspondiente a las mismas pueden hallarse en las siguientes publicaciones: Bahía Lapataia (Borromei \& Quattrocchio, 2007), Río Ovando (Candel et al., 2009), Albufera Lanushuaia (Candel et al., 2011) y Río Varela (Grill et al., 2002). En el presente trabajo no se incluyen los datos de los palinomorfos acuáticos identificados en las localidades de Aserradero Lapataia 1-2 y Arroyo Baliza (Rabassa et al., 2009) debido a la baja densidad del muestreo. El análisis palinológico detallado de dichas secuencias marinas será llevado a cabo próximamente por uno de los autores (M.S.C.).

El tratamiento físico-químico de las muestras fósiles se realizó según las técnicas de Heusser \& Stock (1984), con modificaciones de Dale (1976) para la preservación del paleomicroplancton marino de pared orgánica. Las muestras fueron tratadas con ácidos en frío (HCL y HF) y se omitió la acetólisis y oxidación con la finalidad de preservar el microplancton de pared orgánica. El residuo fue filtrado por tamiz de $10 \mu \mathrm{m}$ para remover las partículas finas y concentrar el material particulado. La determinación sistemática se realizó mediante microscopía de luz transmitida sobre la base de las características morfológicas y morfométricas de los palinomorfos acuáticos con un aumento de x1000. Los recuentos palinológicos se realizaron sobre un mínimo de 200 palinomorfos. Además, para garantizar la presencia de los taxones menos representados, los preparados fueron recorridos en su totalidad. La estimación de las frecuencias relativas (\%) de los palinomorfos terrestres (granos de polen y esporas) y de los palinomorfos acuáticos (dinoquistes, acritarcos, huevos de copépodos, foraminíferos, algas) se calculó sobre el total de palinomorfos contados. Las muestras palinológicas se encuentran archivadas en el Laboratorio de Palinología, Universidad Nacional del Sur, bajo la denominación UNSP seguido por las siglas correspondientes a la sección estudiada: BL (Bahía Lapataia), RO (Río Ovando), AL (Albufera Lanushuaia) y RV (Río Varela).

La nomenclatura taxonómica de los quistes de dinoflagelados utilizada en este trabajo corresponde a Rochon et al. (1999), Head et al. (2001) y Zonneveld (1997). Los taxones de quistes de dinoflagelados aquí estudiados han sido citados siguiendo a Fensome \& Williams (2004). La asignación de los taxones de dinoquistes fósiles a nivel específico fue efectuada por comparación con formas modernas halladas en las muestras superficiales del fondo del Canal Beagle (Candel, 2010; Candel et al., 2012). Algunos taxones fueron agrupados juntos cuando no pudieron identificarse claramente a nivel de especie, debido a la orientación de los ejemplares y/o pobre preservación del material. Este es el caso de Brigantedinium spp. donde se agruparon ejemplares de Brigantedinium simplex y Brigantedinium cariacoense. De la misma manera, Echinidinium spp. comprende a Echinidinium granulatum, Echinidinium cf. E. granulatum, y Echinidinium cf. E. delicatum. Dentro de Spiniferites spp., se incluyen Spiniferites ramosus, Spiniferites mirabilis y aquellos ejemplares que muestran una afinidad con el género Spiniferites. Las algas correspondientes a Zygnemataceae (Spirogyra y Zygnema) y Botryococcaceae (Botryococcus), son agrupadas en "algas dulceacuícolas" mientras que las algas pertenecientes a Prasinophyceae (Cymathiosphaeraceae, Tasmanaceae) se incluyen dentro de "algas marino-salobres". Dentro del grupo Zoomorfos se mencionan los huevos de copépodos y revestimientos de foraminíferos identificados en las muestras estudiadas, aunque las descripciones taxonómicas de los mismos no son incluidas en el presente trabajo.

Los taxones asignados a nivel especifico son mencionados pero no son descriptos en el presente trabajo (ver Candel, 2010; Candel et al., 2012), por el contrario, se incluyen las descripciones taxonómicas de aquellos ejemplares de microplancton de pared orgánica con asignación abierta. Los taxones identificados para cada localidad estudiada se muestran en la Tabla 1. Las características paleoecológicas de los dinoquistes marinos identificados en el material estudiado se presentan en la Tabla 2.

Con el fin de correlacionar las distintas localidades marinas fósiles estudiadas en el Canal Beagle, se utilizaron los fechados radiocarbónicos realizados sobre dichos depósitos sin calibrar (Tabla 3). Se debe considerar un efecto reservorio de $556 \pm 61$ años, según lo analizado por Albero et al. (1988) para el área del Canal Beagle. Las dataciones radiocarbónicas fueron realizadas sobre materia orgánica y valvas de moluscos en el CSIR Environmentek, Pretoria, Sudáfrica y AMS Facility, University of Arizona, Tucson, U.S.A. 
Tabla 1. Listado de dinoquistes y restantes palinomorfos acuáticos identificados en cada localidad estudiada. Abreviaturas: BL, Bahía Lapataia (Borromei \& Quattrocchio, 2007); RV, Río Varela (Grill et al., 2002); AL, Albufera Lanushuaia (Candel et al., 2011); RO, Río Ovando (Candel et al., 2009).

Table 1. List of identifified dinocyst and other aquatic palynomophs for each studied locality. Abbreviations: BL, Bahía Lapataia (Borromei \& Quattrocchio, 2007); RV, Río Varela (Grill et al., 2002); AL, Albufera Lanushuaia (Candel et al., 2011); RO, Río Ovando (Candel et al., 2009).

\begin{tabular}{|c|c|c|c|c|}
\hline Localidades & $\mathrm{BL}$ & $\mathrm{RV}$ & $\mathrm{AL}$ & $\mathrm{RO}$ \\
\hline Edad años $\mathrm{C}^{14} \mathrm{AP}$ & $8.200-? 5.500$ & $6.200-6.000$ & $6.000-? 5.000$ & $4.200-3.500$ \\
\hline Paleoambientes & \multicolumn{2}{|c|}{$\begin{array}{l}\text { Nerítico interno a externo } \\
\text { Salinidad: baja y variable } \\
\text { Temperatura del agua: templado-fría. Abundancia } \\
\text { de nutrientes disueltos por aporte de agua dulce por } \\
\text { derretimiento glacial }\end{array}$} & \multicolumn{2}{|c|}{$\begin{array}{l}\text { Nerítico interno a marino marginal } \\
\text { Salinidad: baja a moderada. } \\
\text { Temperatura del agua: templado-fría. Alta } \\
\text { concentración de nutrientes en aguas marinas } \\
\text { superficiales por aporte por escorrentía superficial }\end{array}$} \\
\hline
\end{tabular}

\section{Dinoquistes}

Peridiniales

Brigantedinium simplex

Brigantedinium spp.

Dubridinium cf. D. sp.

Echinidinium cf. E. delicatum

Echinidinium granulatum

Echinidinium cf. E. granulatum

Echinidinium spp.

Islandinium minutum

Pentapharsodinium cf. $P$. dalei

Selenopemphix nephroides

Selenopemphix quanta

Selenopemphix cf. S. quanta

Selenopemphix sp.

Votadinium spinosum

$\mathrm{X}$

$\mathrm{X}$

$\mathrm{x}$

$x-1+2$

$\mathrm{X}$

$\mathrm{X}$

$\mathrm{X}$

$\mathrm{X}$

$\mathrm{X}$

$\mathrm{X}$

\section{Gonyaulacales}

Operculodinium centrocarpum

Operculodinium cf. O. centrocarpum

Spiniferites sp.

Spiniferites spp.

Gymnodiniales

Polykrikos kofoidii

Polykrikos schwartzii

\section{Acritarcos}

Acritarco sp. 1

Halodinium sp.

Michrystridium sp.

Sigmopollis sp.

\section{Otras algas}

Botryococcus braunii

Cymatiosphaera sp.

Spirogyra sp.

Tasmanites sp.

Zygnema sp. 
Tabla 2. Características paleoecológicas de los dinoquistes marinos identificados en las localidades estudiadas. Rangos de temperatura $\left(T^{\circ} \mathrm{C}\right)$, salinidad (ups) y características paleoambientales.

Table 2. Paleoecological characteristics of marine dinocysts identified in the study areas. Temperature $\left(\mathrm{T}^{\circ} \mathrm{C}\right)$ and salinity (psu) ranges, and paleoenvironmental characteristics.

\begin{tabular}{|c|c|c|c|}
\hline Dinoquistes marinos & Temperatura ${ }^{\circ} \mathrm{C}$ & $\begin{array}{l}\text { Salinidad } \\
\text { (ups) }\end{array}$ & Características paleoecológicas \\
\hline \multicolumn{4}{|l|}{ Peridiniales } \\
\hline Brigantedinium simplex & $9-14$ & $<30$ & Distribución nerítica interna, en zonas templado-frías y polares \\
\hline Brigantedinium spp. & $-2,1-29,6$ & $17-36,8$ & Ambientes costeros a oceánicos \\
\hline Dubridinium cf. $D$. sp. & $16-21$ & $>36$ & Ambientes marinos de bajas latitudes \\
\hline Echinidinium cf. E. delicatum & $13,4-29,6$ & $26,5-36,6$ & Áreas con concentraciones relativamente altas de nutrientes en las aguas superficiales \\
\hline Echinidinium granulatum & $13,4-29,6$ & $24,7-36,6$ & Áreas de "upwelling" y regiones influenciadas por la descarga de ríos \\
\hline Islandinium minutum & $-2,1-27$ & $21,3-35,3$ & Ambientes estuarinos, preferentemente en zonas subpolares a polares \\
\hline Pentapharsodinium cf. P. dalei & $-2,1-29,6$ & $21,3-36,7$ & Ambientes costeros a marinos profundos, preferentemente templados fríos a subpolares \\
\hline Selenopemphix nephroides & $1-29,6$ & $>28,5$ & Ambientes costeros, pero no restringido a ellos, en regiones templadas a tropicales \\
\hline Selenopemphix quanta & $-2,1-29,6$ & $16,9-36,7$ & Zonas costeras, de regiones polares a subtropicales \\
\hline Votadinium spinosum & $12,2-27,8$ & $>31,7$ & Ambientes completamente marinos, templados a subtropicales \\
\hline \multicolumn{4}{|l|}{ Gonyaulacales } \\
\hline $\begin{array}{l}\text { Operculodinium } \\
\text { centrocarpum }\end{array}$ & $-2,1-29,6$ & $16,1-36,8$ & Zonas costeras y oceánicas \\
\hline Spiniferites sp. & $13-20$ & $<36$ & Ambientes neríticos internos a externos, en regiones templadas a tropicales \\
\hline \multicolumn{4}{|l|}{ Gymnodiniales } \\
\hline Polykrikos schwartzii & $25-29,1$ & $31,6-36,6$ & Regiones costeras (plataforma) subtropicales/tropicales y zonas de "upwelling" \\
\hline Polykrikos kofoidii & $-0,9-27,5$ & $>28,5$ & Ambientes costeros a oceánicos abiertos, en regiones templado-frías a subtropicales. \\
\hline
\end{tabular}

Tabla 3. Fechados radiocarbónicos publicados de las terrazas marinas, Canal Beagle (edades convencionales, localidades, altitud ( $\mathrm{m}$ s.n.m.) y profundidad de las muestras según los distintos autores.

Table 3. Published radiocarbon dates from marine terraces, Beagle Channel (conventional ages, localities, altitudes ( $\mathrm{m}$ a.s.I) and samples depth as reported different authors.

\begin{tabular}{|c|c|c|c|c|c|}
\hline Localidad (m s.n.m.) & Profundidad $(\mathrm{cm})$ & $\mathrm{N}^{\circ}$ Laboratorio & $\mathrm{Años} \mathrm{C}^{14} \mathrm{AP}$ & Material & Autores \\
\hline \multirow{3}{*}{ Río Ovando (2.5 m) } & $24-26$ & AA75400 & $3.542+38$ & materia orgánica & Candel et al. (2009) \\
\hline & $58-60$ & AA69513 & $4.064+35$ & materia orgánica & Candel et al. (2009) \\
\hline & $70-72$ & Pta7573 & $4.160+45$ & valvas & Coronato et al. (1999) \\
\hline \multirow{3}{*}{ Albufera Lanushuaia (¿?) } & 3 & AA86258 & Post-bomba & materia orgánica & Candel et al. (2011) \\
\hline & 37 & Pta8523 & $5.560 \pm 110$ & materia orgánica & Candel et al. (2011) \\
\hline & 71 & Pta 8526 & $5.750 \pm 80$ & valvas & Candel et al. (2011) \\
\hline \multirow{2}{*}{ Río Varela $(3,0$ m) } & 140 & Pta7606 & $6.060 \pm 70$ & madera & Grill et al. (2002) \\
\hline & 175 & Pta7581 & $6.240 \pm 70$ & valvas & Coronato et al. (1999) \\
\hline Lago Roca (3,95 m) & ¿? & NZ7730 & $7.518 \pm 58$ & valvas & Gordillo et al. (1993) \\
\hline Bahía Lapataia 2 (1,95 m) & 57 & SI6739 & $5.800 \pm 65$ & valvas & Rabassa et al. (1986) \\
\hline Bahía Lapataia 1 & 45 & SI6738 & $7.260 \pm 70$ & valvas & Rabassa et al. (1986) \\
\hline$(1,65 \mathrm{~m})$ & 62 & SI6737 & $8.240 \pm 60$ & valvas & Rabassa et al. (1986) \\
\hline
\end{tabular}

\section{SISTEMÁTICA PALINOLÓGICA}

Acontinuación se detallan los ejemplares microplanctónicos taxonómicamente identificados y se describen aquellos ejemplares con asignación abierta a nivel específico.

División DINOFLAGELLATA (Bütschli, 1885) Fensome et al., 1993
Subdivisión DINOKARYOTA Fensome et al., 1993 Clase DINOPHYCEAE Pascher, 1914 Subclase PERIDINIPHYCIDAE Fensome et al., 1993 Orden PERIDINIALES Haeckel, 1894 Suborden PERIDININEAE (autonimia) Familia PROTOPERIDINIACEAE Balech, 1988 Subfamilia PROTOPERIDINIOIDAE Balech, 1988 
Brigantedinium Reid, 1977 ex Lentin \& William, 1993

Especie tipo. Brigantedinium simplex Wall, 1965 ex Lentin \& William, 1993.

Brigantedinium simplex Wall, 1965 ex Lentin \& William, 1993 (Figuras 2A-B)

Dimensiones. Diámetro máximo: 42,4 $\mu \mathrm{m}$ (1 ejemplar medido). Material estudiado. UNSP 2037: Q28.

Islandinium (Harland \& Reid en Harland et al., 1980) Head et al., 2001

Especie tipo. Algidasphaeridium capillatum Matsuoka \& Bujak, 1988.

Islandinium minutum (Harland \& Reid en Harland et al., 1980) Head et al., 2001

(Figura 2H)

Dimensiones. Diámetro máximo del cuerpo central: 25(28,8)-33 $\mu \mathrm{m}$. Longitud de los procesos: 3-(4,3)-5,5 $\mu \mathrm{m}$, (8 ejemplares medidos).

Material estudiado. UNSP 1968: C29/2, G38, S15, B51/3, S14/2; UNSP 1966: J44, Y31/1; UNSP 2587: R44.

Echinidinium Zonneveld, 1997 ex Head et al., 2001

Especie tipo. Echinidinium granulatum Zonneveld, 1997.

Echinidinium granulatum Zonneveld, 1997

(Figura 2F)

Dimensiones. Diámetro del cuerpo central: 22-(27,4)-35 $\mu \mathrm{m}$. Longitud de los procesos: 3,5-(6)-10 $\mu \mathrm{m}$, (6 ejemplares medidos).

Material estudiado. UNSP 1968: F46/2, C17/1; UNSP 1966: P53/4, W59, T31/2; UNSP 2637: T56/3.

Echinidinium cf. E. granulatum Zonneveld, 1997

(Figura 2G)

Descripción. Quiste corado de cuerpo esferoidal con numerosos procesos no tabulares. La superficie del perifragma es granulada y de color marrón claro. Los procesos son acuminados, huecos y sólidos, cuyas bases son circulares y expandidas. Arqueopilo indicado por un "split" simple a lo largo de una o más suturas.

Dimensiones. Diámetro del cuerpo central: 21-(29,3)-43 $\mu \mathrm{m}$. Longitud de los procesos: 4-(6,1)-9 $\mu \mathrm{m}$. Diámetro de las bases de los procesos: 1-(1,6)-3 $\mu \mathrm{m}$ (3 ejemplares medidos). Material estudiado. UNSP 1968: X12/2, W23/1; UNSP 1966: W49/4.

Observaciones. Difieren de E. granulatum por tener procesos más cortos que los definidos por Zonneveld (1997) y de ambos tipos: huecos y sólidos. Por esta razón se prefiere comparar y no asignar a Echinidinium granulatum.
Echinidinium cf. E. delicatum Zonneveld, 1997

(Figura 2E)

Descripción. Quiste próximo-corado de contorno circular con numerosos y delgados procesos. La superficie del perifragma es lisa y de color marrón pálido. Procesos no tabulares, huecos y acuminados, con bases sub-circulares en el corte transversal. El arqueopilo indicado por un "split" a lo largo de una o más suturas. Dimensiones. Diámetro de cuerpo: 19-(22,5)-28 $\mu \mathrm{m}$. Longitud de los procesos: 2,5-(4,2)- $6 \mu \mathrm{m}$ (4 ejemplares medidos).

Material estudiado. UNSP 1968: Q25, E32/1; UNSP 1966: Q34/2; UNSP 2033: Q34.

Observaciones. En los ejemplares estudiados se reconocieron procesos huecos y acuminados tal como describe Zonneveld (1997) pero no pudo observarse claramente la pared del quiste debido a una mala preservación, por lo tanto se ha preferido su comparación con Echinidinium delicatum.

Selenopemphix Benedek, 1972 emend. Head, 1993

Especie tipo. Selenopemphix nephroides Benedek, 1972 emend. Benedek \& Sarjeant, 1981.

Selenopemphix nephroides Benedek, 1972 emend. Benedek \& Sarjeant, 1981

(Figura 2I)

Dimensiones. Diámetro máximo del cuerpo: 42,4-(56)-63 $\mu \mathrm{m}$, (3 ejemplares medidos).

Material estudiado. UNSP 1631a: C22/2; UNSP 2588: N18; UNSP 2036: M49.

Observaciones. Los ejemplares medidos, a excepción de uno de ellos, muestran un tamaño levemente mayor del cuerpo que aquellos ejemplares descriptos previamente por otros autores (Head, 1993; Rochon et al., 1999; Marret \& Zonneveld, 2003).

Selenopemphix quanta (Bradford, 1975) Matsuoka, 1985

(Figura 2J)

Dimensiones. Diámetro (ancho) del cuerpo central: 44,1(53,5)-63 $\mu \mathrm{m}$. Longitud de los procesos: 8-(9,2)-10,5 $\mu \mathrm{m},(2$ ejemplares medidos).

Material estudiado. UNSP 2636: G26/3; UNSP 1972b: M38/2.

$$
\begin{gathered}
\text { Selenopemphix cf. S. quanta (Bradford, 1975) } \\
\text { Matsuoka, 1985 } \\
\text { (Figura 2K) }
\end{gathered}
$$

Descripción. Quiste con compresión polar de forma subesférica, cuya pared es lisa de color marrón. Procesos sólidos, acuminados y truncados, con bases levemente expandidas y homogéneamente distribuidos. El arqueopilo intercalar (2a) no fue claramente observado en los ejemplares estudiados.

Dimensiones. Diámetro (ancho) del cuerpo central: $38 \mu \mathrm{m}$. Longitud máxima de los procesos: $8 \mu \mathrm{m}$ (1 ejemplar medido). 
Material estudiado. UNSP 2637: 049/4.

Observaciones. Este ejemplar se compara y no se asigna a Selenopemphix quanta por registrar un diámetro menor del cuerpo central (S. quanta: 40-83 $\mu \mathrm{m}$, Rochon et al., 1999), baja densidad y menor longitud de los procesos ( $S$. quanta: 10-16 $\mu \mathrm{m}$, Rochon et al., 1999) sin bases expandidas.

\section{Selenopemphix sp.}

(Figura 2L)

Descripción. Quiste con compresión polar, de forma ovoidal o reniforme. Pared lisa, de color amarillo pálido. Cíngulo indicado por un borde levemente más oscuro mostrando una marcada indentación sulcal. Arqueopilo no claramente observable.

Dimensiones. Diámetro máximo del cuerpo: $48 \mu \mathrm{m}$, (1 ejemplar medido).

Material estudiado. UNSP 2037: G39/1.

Observaciones. El ejemplar estudiado difiere de $S$. nephroides por presentar la pared del quiste más delgada y de un color muy pálido, posiblemente relacionado con la preservación del material. Asimismo, este ejemplar muestra características morfológicas comparables con $S$. antarctica descripto por Marret \& de Vernal (1997), aunque no pudo observarse claramente la ornamentación granular concentrada alrededor de los cuernos apical y antapicales, rasgo característico de $S$. antarctica. Dada la escasez del presente material se prefiere mantener asignación abierta para el mismo.

Votadinium Reid, 1977

Especie tipo. Votadinium calvum Reid, 1977.

Votadinium spinosum Reid, 1977

(Figura 2M)

Dimensiones. Ancho del cuerpo central: $70 \mu \mathrm{m}$. Longitud del cuerpo central: $65 \mu \mathrm{m}$. Longitud de las espinas: $5 \mu \mathrm{m}$, (1 ejemplar medido).

Material estudiado. UNSP 2638: Q47/4.

Observaciones. El ejemplar medido presenta dimensiones mayores del cuerpo central que el descripto por otros autores ( V. spinosum, ancho: 54-63 $\mu \mathrm{m}$; longitud: $49-54 \mu \mathrm{m}$, Marret \& Zonneveld, 2003).

Subfamilia DIPLOPSALIOIDAE Abé, 1981

Dubridinium Reid, 1977

Especie tipo. Dubridinium cavatum Reid, 1977.

\section{Dubridinium cf. D. sp.}

(Figuras 2C-D)

Descripción. Quiste proximado de contorno sub-circular y color marrón oscuro. Pared compuesta por dos capas, endofragma granulado y perifragma liso. Arqueopilo no discernible. Se observa la cicatriz flagelar.
Dimensiones. Diámetro máximo del cuerpo: 46,4-(47,6)-48,8 $\mu \mathrm{m}$ (2 ejemplares medidos).

Material estudiado. UNSP 2582: K50/4; UNSP 2579: U42/2.

Observaciones. Los ejemplares estudiados son morfológicamente similares a la especie tipo pero no son asignados por presentar deformación del cuerpo del quiste.

Familia PERIDINIACEAE Ehrenberg, 1831 Subfamilia CALCIODINELLOIDEAE

Fensome et al., 1993

\section{Pentapharsodinium Indelicato \& Loeblich III, 1986 emend. Montresor et al., 1993}

Especie tipo. Pentapharsodinium dalei Indelicato \& Loeblich III, 1986.

\section{Pentapharsodinium cf. P. dalei}

(Figura 2N)

Descripción. Quiste próximo-corado y cuerpo central de contorno circular. Superficie de la pared lisa y hialina, con una densidad variable de procesos no tabulares, sólidos, bifurcados en los extremos y de bases expandidas. Arqueopilo indicado por una hendidura (tipo chásmico).

Dimensiones. Diámetro del cuerpo central: $26 \mu \mathrm{m}$. Longitud de los procesos: 5,6 $\mu \mathrm{m}$, (1 ejemplar medido).

Material estudiado. UNSP 2032: S22/4.

Observaciones. El presente ejemplar es comparado y no asignado $P$. dalei debido a una mala preservación del material estudiado.

Orden GONYAULACALES Taylor, 1980

Suborden GONYAULACINEAE (autonimia)

Familia GONYAULACACEAE Lindemann, 1928

Subfamilia CRIBOPERIDINIOIDEAE Fensome et al., 1993

Operculodinium Wall, 1967 emend. Matsuoka et al., 1997

Especie tipo. Operculodinium centrocarpum Deflandre \& Cookson 1955 sensu Wall, 1967.

Operculodinium centrocarpum

Deflandre \& Cookson 1955 sensu Wall, 1967

(Figura 2O)

Dimensiones. Diámetro del cuerpo: 50,4-(52,5)-54,6 $\mu \mathrm{m}$. Longitud de los procesos: 8-(9)-10 $\mu \mathrm{m}$, (2 ejemplares medidos). Material estudiado. UNSP 1532a: F23/3; UNSP 834a: V32. Observaciones. Los ejemplares estudiados presentan un diámetro mayor del cuerpo central que los descriptos por otros autores (Rochon et al., 1999; Marret \& Zonneveld, 2003).

Operculodinium cf. O. centrocarpum

Deflandre \& Cookson 1955 sensu Wall, 1967

(Figura 2P) 
Descripción. Quiste corado de cuerpo esferoidal, con numerosos procesos delgados, no tabulares El perifragma es delgado, hialino y la superficie es microgranulada. Los procesos son huecos, abiertos distalmente y con bases circulares. El arqueopilo no es claramente observado.

Dimensiones. Diámetro máximo del cuerpo: $56 \mu \mathrm{m}$. Longitud de los procesos: 9,6 $\mu \mathrm{m}$ (1 ejemplar medido).

Material estudiado. UNSP 1972b: C14/4.

Observaciones. Se encontraron ejemplares de $O$. centrocarpum en Bahía Lapataia y Río Varela, mientras que los ejemplares hallados en la sección de Río Ovando presentan deformación y/o mala preservación. Por este motivo no son asignados y se prefiere su comparación con $O$. centrocarpum.

\section{Subfamilia GONYAULACOIDEAE (autonimia)}

Spiniferites Mantell, 1850 emend. Sarjeant, 1970

Especie tipo. Spiniferites ramosus (Ehrenberg 1838) Loeblich \& Loeblich, 1966.

Spiniferites sp.

(Figura 2Q)

Descripción. Quiste próximo-corado de forma ovoidal y procesos gonales. Pared microgranulada y color amarillo pálido. Los procesos son bi- o trifurcados. En el área antapical se observan procesos conectados por una membrana. El arqueopilo no se observó.

Dimensiones. Longitud del cuerpo central: 42-(56,7)$75,6 \mu \mathrm{m}$. Longitud de los procesos: 10-(13,3)-16,8 $\mu \mathrm{m}$ (4 ejemplares medidos).

Material estudiado. UNSP 835a: Q24; UNSP 843a: K53; UNSP 853b: V43/44; UNSP 1629c: X45/2.

Observaciones. Estos ejemplares presentan características morfológicas similares a Spiniferites mirabilis (Rossignol, 1964) Sarjeant, 1970, sin embargo se mantiene asignación abierta de los mismos por presentar un mal estado de preservación.

\section{Subclase GYMNODINIPHYCIDAE Fensome et al., 1993 Orden GYMNODINIALES Apstein, 1909 \\ Suborden GYMNODINIINEAE (autonimia) \\ Familia POLYKRIKACEAE Kofoid \& Swezy, 1921}

\section{Polykrikos Bütschli, 1873}

Comentario. Género basado en la teca móvil.

Polykrikos kofoidii Chatton, 1914

(Figura 2R)

Dimensiones. Longitud del cuerpo del quiste: 63-(71,4)$79,8 \mu \mathrm{m}$. Longitud de la ornamentación: 5-(7,7)-10,5 $\mu \mathrm{m},(2$ ejemplares medidos).

Material estudiado. UNSP 1972b: B50; UNSP 2638: Y27

Polykrikos schwartzii Bütschli, 1873

(Figuras 2S-T)
Dimensiones. Longitud del cuerpo del quiste: 40-(47)-55 $\mu \mathrm{m}$. Longitud de los procesos: 8-(9)-10 $\mu \mathrm{m}$ (3 ejemplares medidos).

Material estudiado. UNSP 1972b: F37/1, F47/3; UNSP 1972c: J50/1.

Observaciones. Polykrikos schwartzii ha sido previamente documentado como Polykrikos kofoidii (por ej., Matsuoka, 1985, 1987; Marret \& Zonneveld, 2003; Radi \& de Vernal, 2004). En el presente trabajo, la identificación de ambas especies $(P$. schwartzii y $P$. kofoidii) se ajusta con la presentada por Matsuoka et al., 2009. Algunos ejemplares difieren del típico quiste de Polykrikos schwartzii por presentar un tamaño menor del cuerpo (longitud del cuerpo: 52,5-74,9 $\mu \mathrm{m}$; longitud de los procesos: 11,8-17,9 $\mu \mathrm{m}$, como P. kofoidii en Matsuoka, 1985).

\section{Grupo ACRITARCHA Evitt, 1963}

Halodinium Bujak, 1984

Especie tipo. Halodinium major Bujak, 1984.

\section{Halodinium sp.}

(Figura 3A)

Descripción. Palinomorfo discoidal aplanado. La pared del cuerpo es lisa a microgranulada, delgada y hialina. La membrana externa se extiende alrededor del cuerpo central, es delgada y a menudo se presenta incompleta por rotura. El piloma central es circular con un borde liso bien definido, usualmente engrosado. Dimensiones. Diámetro del cuerpo central (excluyendo la membrana): 38,4-(66,7)-92 $\mu \mathrm{m}$. Diámetro del piloma: 9,6(17)-39,9 $\mu \mathrm{m}$ (13 ejemplares medidos).

Material estudiado. UNSP 1970: W44; UNSP 1969: J52/2; UNSP 1968: U37, J44/1; UNSP 2034: R60, K44/2; UNSP 2033: C33; UNSP 2103: P19; UNSP 2588: P16/2; UNSP 2586: H36; UNSP 2581: K56/2; UNSP 2580: A47, D14/2.

Observaciones. Los ejemplares estudiados corresponden al género Halodinium, aunque presentan medidas con valores variables que dificultan su asignación a nivel especifico dentro de los rangos correspondientes a $H$. major y $H$. minor.

Micrhystridium Deflandre, 1937 emend. Sarjeant \& Stancliffe, 1994

Especie tipo. Micrhystridium inconspicuum (Deflandre, 1935) Deflandre 1937 emend. Deflandre \& Sarjeant 1970.

\section{Micrhystridium sp.}

(Figura 3B)

Descripción. Vesícula esferoidal de pared hialina y delgada con espinas cortas, delgadas y rectas, regularmente distribuidas. En algunos ejemplares el piloma esta representado por pequeñas hendiduras rectas ("slit") o por formas poligonales. Dimensiones. Diámetro del cuerpo central: 16,8-(19)-25 $\mu \mathrm{m}$. Longitud de las espinas: 2,1-(3,3)-4 $\mu \mathrm{m}$ (5 ejemplares medidos). Material estudiado. UNSP 1532a: C34; UNSP 1969: P51; UNSP 1966: N50/1; UNSP 2036: L51/1; UNSP 2102: N57. 

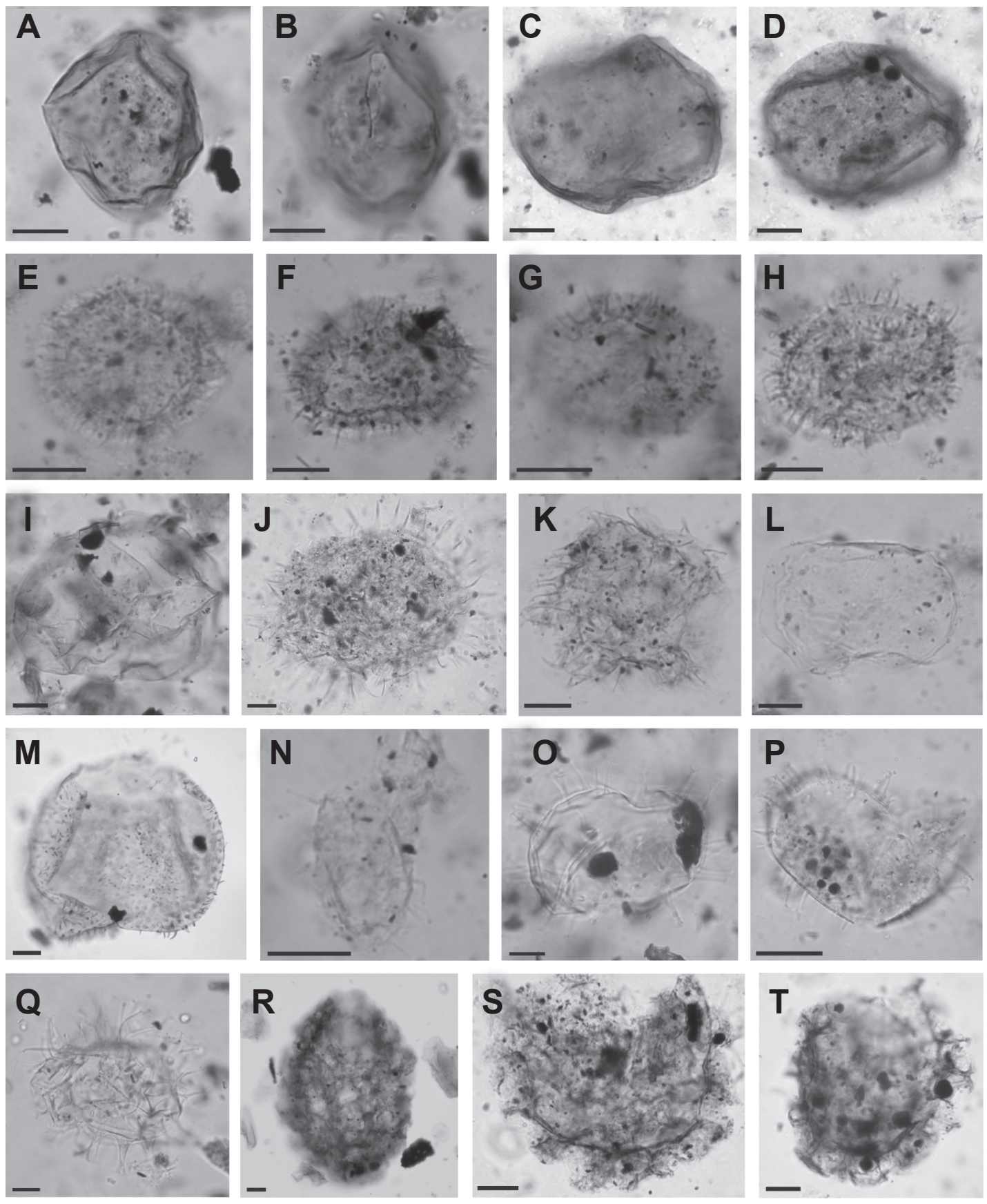

Figura 2. Quistes de dinoflagelados identificados en los sedimentos fósiles del Canal Beagle. Número de muestra seguido por coordenadas England Finder. A-B, Brigantedinium simplex (UNSP RO2037: Q28/4), A, vista dorsal, foco bajo; B, vista dorsal, foco alto. C-D, Dubridinium sp. (UNSP AL2582: K50/4), C, foco bajo; D, foco alto. E, Echinidinium cf. E. delicatum (UNSP R01968: Q25). F, Echinidinium granulatum (UNSP RO1968: F46/2). G, Echinidinium cf. E. granulatum (UNSP RO1968: X12/2). H, Islandinium minutum (UNSP RO1968: S14/2). I, Selenopemphix nephroides (UNSP BL1631a: C22/2). J, Selenopemphix quanta (UNSP RO1972b: M38/2). K, Selenopemphix cf. S. quanta (UNSPAL2637: O49/4). L, Selenopemphix sp. (UNSP RO2037: G39/1). M, Votadinium spinosum (UNSP AL2638: Q47/4). N, Pentapharsodinium cf. P. dalei (UNSP RO2032: S22/4). O, Operculodinium centrocarpum (UNSP RV1532a: F23/3). P, Operculodinium cf. O. centrocarpum (UNSP RO: XX). Q, Spiniferites sp. (UNSP BL843a: K53). R, Polykrikos kofoidii (UNSP AL2638: Y27). S-T, Polykrikos schwartzii; S (UNSP RO 1972b: F37/1); T (UNSP RO1972c: J50/1). Escalas = $10 \mu \mathrm{m}$.

Figure 2. Dinoflagellate cysts identified in fossil sediments from Beagle Channel. Number of sample followed by England Finder coordinates. A-B, Brigantedinium simplex (UNSP RO2037: Q28/4), A, dorsal view, low focus; B, dorsal view, high focus. C-D, Dubridinium sp. (UNSP AL2582: K50/4), C, low focus; D, high focus. E, Echinidinium cf. E. delicatum (UNSP RO1968: Q25). F, Echinidinium granulatum (UNSP RO1968: F46/2). G, Echinidinium cf. E. granulatum (UNSP RO1968: X12/2). H, Islandinium minutum (UNSP RO1968: S14/2). I, Selenopemphix nephroides (UNSP BL1631a: C22/2). J, Selenopemphix quanta (UNSP RO1972b: M38/2). K, Selenopemphix cf. S. quanta (UNSP AL2637: O49/4). L, Selenopemphix sp. (UNSP RO2037: G39/1). M, Votadinium spinosum (UNSP AL2638: Q47/4). N, Pentapharsodinium cf. P. dalei (UNSP RO2032: S22/4). O, Operculodinium centrocarpum (UNSP RV1532a: F23/3). P, Operculodinium cf. O. centrocarpum (UNSP RO: XX). Q, Spiniferites sp. (UNSP BL843a: K53). R, Polykrikos kofoidii (UNSP AL2638: Y27). S-T, Polykrikos schwartzii; S (UNSP RO 1972b: F37/1); T (UNSP RO1972c: J50/1). Scale bars $=10 \mu \mathrm{m}$. 
Observaciones. Los ejemplares estudiados presentan características morfológicas similares a la especie tipo, aunque se observa una variabilidad en el tamaño de la vesícula y longitud de las espinas. Por este motivo se mantiene asignación abierta para la especie.

\section{Sigmopollis Hedlund, 1965}

Especie tipo. Sigmopollis hispidus Hedlund, 1965.

Sigmopollis sp.

(Figuras 3C-D)

Descripción. Microfósiles globosos de pared lisa con una apertura en forma sigmoidea.

Dimensiones. Diámetro máximo del cuerpo: 14,7-(16,8)-21 $\mu \mathrm{m}$ (6 ejemplares medidos).

Material estudiado. UNSP 1968: T41, T40/2, T54/3, T56/2, $\mathrm{R} 43, \mathrm{X} 43$.

Observaciones. Estos ejemplares difieren de Sigmopollis hispidus en la ausencia de espinas que caracterizan la especie tipo. Asimismo, estas formas son similares a los microfósiles (Tipo 128) descriptos por Pals et al. (1980) y van Geel et al. (1983), diferenciándose de estos últimos en presentar un tamaño menor y pared lisa del cuerpo.

\section{Acritarco sp. 1}

(Figuras 3E-F)

Descripción. Palinomorfo de contorno sub-circular, pared gruesa, psilada a microgranulada y pliegues concéntricos. Presenta un piloma circular de contorno bien definido y, en algunos casos se observa una membrana muy delgada rodeando el cuerpo.

Dimensiones. Diámetro del cuerpo central (excluyendo membrana): 62,4-(69,1)-82 $\mu \mathrm{m}$. Diámetro del piloma: 29,6(31) $-32 \mu \mathrm{m}$ (3 ejemplares medidos).

Material estudiado. UNSP 2584: V53/3, L56/1, T30/4.

Observaciones. Usualmente se observa el opérculo del piloma en el interior del cuerpo. Para lograr una asignación sistemática correcta de estas formas es necesario el estudio de un mayor número de ejemplares.

División CHLOROPHYTA Pascher, 1914 Clase TREBOUXIOPHYCEAE Friedl, 1995 Familia BOTRYOCOCCACEAE Wille, 1909

\section{Botryococcus Kützing, 1849}

Especie tipo. Brotryococcus braunii Kützing, 1849.

$$
\text { Botryococcus braunii Kützing, } 1849
$$

(Figura 3G)

Dimensiones. Diámetro máximo de las colonias: $52,5 \mu \mathrm{m}$ (1 ejemplar medido).

Material estudiado. UNSP 1966: Z57/2.

Observaciones. Algunos ejemplares presentaron tendencia a formar una matriz no-estructurada con copas, en secciones transversales, circulares a sub-circulares poco definidas.
División CHAROPHYTA sensu Lewis \& McCourt, 2004

Clase ZYGNEMATOPHYCEAE van den Hoek et al., 1995

Orden ZYGNEMATALES G.M. Smith, 1933

Familia ZYGNEMATACEAE Kützing, 1843

Spirogyra Link, 1820

Especie tipo. Spirogyra porticalis (Müller) Cleve, 1868.

Spirogyra sp.

(Figura 3H)

Descripción. Espora de contorno elíptico (extremos agudos a subredondeados) y pared delgada. La superficie de la pared es lisa, en algunos casos, escabrada. Sutura longitudinal que no rodea completamente la espora permitiendo que las valvas permanezcan unidas al abrirse.

Dimensiones. Diámetro máximo 24-(35,6)-42 $\mu \mathrm{m}$ (14 ejemplares medidos).

Material estudiado. UNSP 1970: O47/2; UNSP 1969: L14/2; UNSP 2099: M49, F51/2, U22; UNSP 2098: Z53, Y51/4, S41/3, C51; UNSP 2579: T40/1; UNSP 1946: N54/1, C48/3; UNSP 1945:Y46, Z27/3.

Observaciones. Los ejemplares estudiados no fueron asignados a nivel específico dado que para ello es necesario analizar rasgos vegetativos-reproductivos, en su mayoría no preservables en el material palinológico fósil. Las esporas estudiadas presentan similitud con aquellas denominadas "Tipo 130" por Pals et al. (1980) y van Geel et al. (1981, 1983), y comparables con Spirogyra sp.1 descripta por Borel et al. (2003). Sin embargo, las dimensiones del presente material no se ajustan perfectamente a los rangos presentados por otros autores.

$$
\text { Zygnema Agardh, } 1816
$$

Especie tipo. Zygnema cruciatum (Vaucher) Agardh, 1816.

Zygnema sp.

(Figuras 3I-J)

Descripción. Esporas de contorno circular a elipsoidal, en algunos casos plegadas sobre sí mismas adoptando una forma de canoa. La pared es lisa, hialina y se caracteriza por presentar una escultura foveolada.

Dimensiones. Diámetro máximo: 54,6-(81,9)-126 $\mu \mathrm{m}$ (6 ejemplares medidos).

Material estudiado. UNSP 2097: U57/1, S48, T36, W45, Q46; UNSP 1947: T49.

Observaciones. Estas esporas presentan características morfológicas similares con aquellas formas descriptas por van Geel \& van der Hammen (1978), van Geel et al. (1981; Tipo 314), Zamaloa (1996), Medeanic (2006). Sin embargo, el material estudiado aquí presenta un rango mayor de tamaño que los previamente citados por otros autores.

División PRASINOPHYTA Round, 1971

Clase PRASINOPHYCEAE Parke en Parke \& Dixon, 1964 Orden PTEROSPERMATALES

Parke \& Green en Parke \& Dixon, 1976 


\author{
Familia TASMANACEAE Sommer 1956 \\ emend. Mädler, 1963
}

Tasmanites Newton, 1875

Especie tipo. Tasmanites punctatus Newton 1875.

Tasmanites sp.

(Figura 3K)

Descripción. Cuerpo de contorno circular y pared gruesa. La superficie de la pared está ornamentada con gránulos sub-circulares hasta poligonales de bajo relieve, distribuidos uniformemente dando el aspecto de un panal de abejas. El centro de cada gránulo es atravesado por un diminuto canal, que en algunos casos no penetra toda la pared. Se observa una hendidura en forma de " $v$ ", posiblemente para la liberación del contenido celular.

Dimensiones. Diámetro máximo del cuerpo: 128,1-130,2 $\mu \mathrm{m}$. Espesor de la pared: 6,3 $\mu \mathrm{m}$ (1 ejemplar medido).

Material estudiado. UNSP 2581: H27/3

Observaciones. De acuerdo con Guy-Ohlson \& Boalch (1992) y Guy-Ohlson (1996), las diferencias y variaciones en el phycoma, tamaño y ornamentación en distintos ejemplares podrían estar indicando diferentes estadíos de desarrollo dentro del ciclo de vida. Ellos sugieren que la presencia de diferentes morfotipos en el material estudiado pueden, o no, pertenecer a una misma especie de acuerdo con la etapa de desarrollo al momento de depositación. Por esta razón no se asigna a nivel específico.

\section{Familia CYMATHIOSPHAERACEAE Mädler, 1963}

Cymatiosphaera (Wetzel, 1933) Deflandre, 1954

Especie tipo. Cymatiosphaera radiata Wetzel, 1933 emend. Sarjeant 1985.

\section{Cymatiosphaera sp.}

(Figuras 3. L-N)

Descripción. Vesícula esferoidal y pared lisa. La superficie externa está dividida por una membrana delgada, sinuosa, levemente elevada formando campos poligonales irregulares. Dimensiones. Diámetro máximo del cuerpo central: 19,2$(29,5)-37,8 \mu \mathrm{m}$. Altura de la membrana: 3,2-(4,3)-6,3 $\mu \mathrm{m}$ (13 ejemplares medidos).

Material estudiado. UNSP 2100: F61/3, R34/4, Y44/3; UNSP 2099: W49, W52/1; UNSP 2097: V46/3, V48/1, M60/4, L61, M50/1, M50/2, K40, K43/2.

Observaciones. En los ejemplares estudiados se observa variaciones en el tamaño del cuerpo central y en la altura de las membranas. Posiblemente estas variaciones representen sucesivos estadíos de desarrollo de una misma especie. Por otro lado, de Vernal \& Mudie (1989), sugieren que estas variaciones morfológicas indican la presencia de varias especies o morfotipos.

\section{CONSIDERACIONES ECOLÓGICAS Y DISTRIBUCIÓN DEL MICROPLANCTON DE PARED}

Ecología y distribución de los quistes de dinoflagelados

La especie Brigantedinium simplex tiene una distribución nerítica interna en el Atlántico Norte (Harland, 1983) e integra las asociaciones marinas de zonas biogeográficas templadofrías y polares (Tabla 2). Las mayores abundancias relativas de este taxón han sido registradas en latitudes por encima de los $55^{\circ} \mathrm{N}$, representando temperaturas superficiales del agua por debajo de los 9 y $14^{\circ} \mathrm{C}$ en invierno y verano, respectivamente (Dale, 1983; Edwards \& Andrle, 1992; Matthiessen, 1995). En Argentina, B. simplex fue recientemente documentado en la plataforma continental de la provincia de Buenos Aires $\left(38^{\circ} 28^{\prime} \mathrm{S}-57^{\circ} 41^{\prime} \mathrm{O}\right)$ por Vilanova et al. (2008) y en sedimentos marinos modernos y fósiles del Canal Beagle (Candel et al., 2009, 2012). Por otro lado, Islandinium minutum muestra una distribución bipolar en áreas costeras al norte de los $30^{\circ} \mathrm{N}$ y al sur de los $30^{\circ} \mathrm{S}$. Los mapas de distribución de Rochon et al. (1999) y de Vernal et al. (2001), indican una distribución en todo el Ártico y dentro de regiones más templadas del Océano Atlántico Norte. Otros registros de esta especie en sedimentos modernos de Chile (Dale, 1996), el sur del Océano Indico (Marret \& de Vernal, 1997), el Océano Austral (Harland et al., 1998; Harland \& Pudsey, 1999) y sur de Argentina (Candel et $a l ., 2012$ ) indican su presencia dentro de regiones subpolares a polares modernas del Hemisferio Sur. Islandinium minutum ha sido observado dentro de un amplio rango de temperaturas y salinidades, entre $-2,1$ y $27^{\circ} \mathrm{C}$ (invierno y verano) y 21,3 y 35,3 ups (primavera y verano, unidades prácticas de salinidad) respectivamente. Sin embargo, las mayores abundancias relativas $(>40 \%)$ son observadas en rangos de temperaturas y salinidades de -2 a $5^{\circ} \mathrm{C}$ y 17 a 34 , respectivamente (de Vernal et al., 2001). Es una especie eurihalina (con amplia tolerancia a salinidades variables), esto explica la gran abundancia en ambientes estuarinos (Head et al., 2001) (Tabla 2).

Echinidinium delicatum y Echinidinium granulatum han sido registrados en áreas con concentraciones relativamente altas de nutrientes en las aguas superficiales, aunque no en relación directa con áreas de "upwelling" (Zonneveld, 1997; Zonneveld et al., 2001). Además, han sido encontrados en regiones influenciadas por la descarga de los ríos caracterizadas por baja salinidad y estratificación de la columna de agua, como por ejemplo, en la descarga del río Amazonas sobre el Atlántico ecuatorial occidental (Vink et al., 2000; Zonneveld et al., 2001) y en el sector sudeste del Atlántico Sur, en la región de Benguela (Sudáfrica), donde una alta abundancia relativa se ha encontrado en las aguas de descarga del Río Orange y cerca de las células de "upwelling" costero (Zonneveld et al., 2001). También han sido registrados en el Pacífico Norte (Radi \& de Vernal, 2004, 2008; Radi et al., 2007). Se trata de especies ampliamente distribuidas en ambientes subtropicales a tropicales con condiciones meso/eutróficas, dentro de rangos relativamente amplios de temperaturas y salinidades que varían entre 13,4 y $29,6^{\circ} \mathrm{C}$ (invierno-verano) y entre 26,5 y $36,6^{\circ}$ (primavera-verano), 

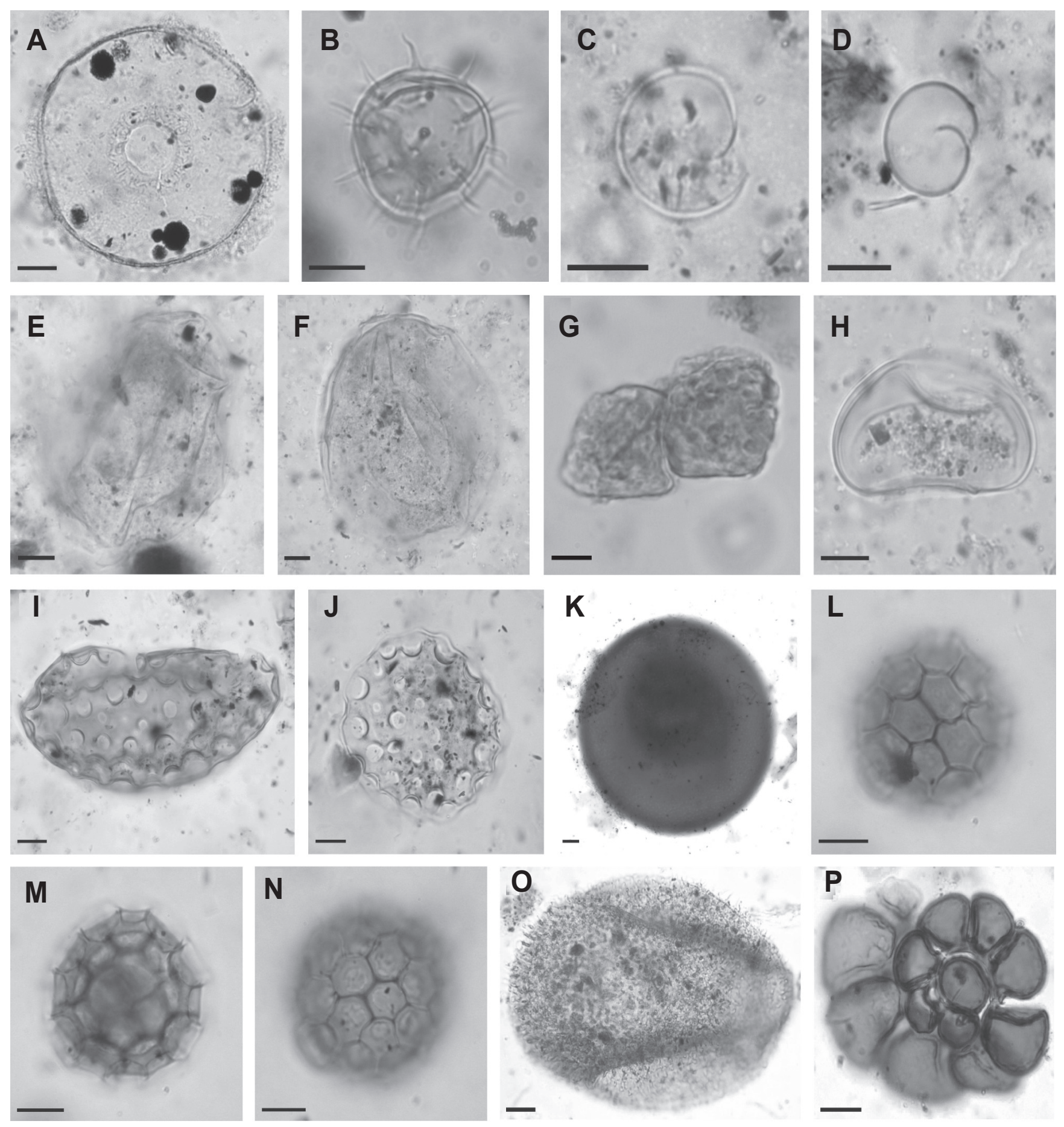

Figura 3. Acritarcos y otros palinomorfos acuáticos identificados en los perfiles marinos estudiados. Número de muestra seguido por coordenadas England Finder. A, Halodinium sp. (UNSP RO1972c: R13). B, Micrhystridium sp. (UNSP BL1532a: C34). C-D, Sigmopollis sp.; C (UNSP RO1968: T40/2); D (UNSP R01968: T41). E-F, Acritarco sp.1; E (UNSP AL 2584: L56/1); F (UNSP AL2584: T30/4). G, Botryococcus braunni (UNSP RO1966: Z57/2). H, Spirogyra sp. (UNSP AL2579: T40/1). I-J, Zygnema sp.; I (UNSP RO2097: U57/1); J (UNSP RO2097: T36). K, Tasmanites sp. (UNSP AL2581: H27/3). L-N, Cymatiosphaera sp.; L-M (UNSP RO2100b: Y44/3); N (UNSP RO2100: F61/3). O, Huevo de copépodo (UNSP RO1972c: G14/2). P, Revestimiento orgánico de foraminífero (UNSP RO1972d: G34/4). Escalas $=10 \mu \mathrm{m}$.

Figure 3. Acritarchs and other aquatic palynomorph identified in the studied marine profiles. Number of sample followed by England Finder coordinates. A, Halodinium sp. (UNSP RO1972c: R13). B, Micrhystridium sp. (UNSP BL1532a: C34). C-D, Sigmopollis sp.; C, (UNSP RO1968: T40/2); D, (UNSP RO1968: T41). E-F, Acritarch sp. 1; E (UNSP AL 2584: L56/1); F (UNSP AL2584: T30/4). G, Botryococcus braunni (UNSP RO1966: Z57/2). H, Spirogyra sp. (UNSP AL2579: T40/1). I-J, Zygnema sp.; I (UNSP RO2097: U57/1); J (UNSP RO2097: T36). K, Tasmanites sp. (UNSP AL2581: H27/3). L-N, Cymatiosphaera sp.; L-M (MUNSP RO2100b: Y44/3); N (UNSP RO2100: F61/3). O, Copepod egg (UNSP RO1972c: G14/2). P, Foraminiferal lining (UNSP RO1972d: G34/4). Scale bars $=10 \mu \mathrm{m}$ 
respectivamente (Marret \& Zonneveld, 2003) (Tabla 2). Sin embargo, el registro de estos taxones en sedimentos modernos y fósiles del Canal Beagle (Candel et al., 2009, 2011, 2012) indican una tolerancia de estas especies a temperaturas más frías que las previamente registradas para otras regiones.

La distribución de Selenopemphix nephroides está restringida a la región comprendida entre $\operatorname{los} 50^{\circ} \mathrm{N}$ y $50^{\circ} \mathrm{S}$, en cercanías del Frente Subtropical del Hemisferio Sur y en áreas completamente marinas influenciadas por la descarga del río Amazonas en el oeste ecuatorial del Océano Atlántico. Puede considerarse una especie completamente marina, templada a tropical, presente en ambientes costeros pero no restringida a ellos (Tabla 2). Se distribuye dentro de un amplio rango de temperaturas $\left(1\right.$ y $29,6^{\circ} \mathrm{C}$; invierno y verano, respectivamente), salinidades superiores a 28,5 y con altas abundancias en sedimentos depositados en ambientes meso a eutróficos (Marret \& Zonneveld, 2003). Este taxón se incluye, al igual que Dubridinium sp., dentro de un grupo de protoperidiniodeos que tienen rara ocurrencia en latitudes medias a altas y que están presentes en muy bajo porcentaje en las muestras estudiadas y solamente en un $10 \%$ de las muestras de la base de datos mundial. En su mayoría han sido registrados en latitudes bajas del Océano Atlántico Norte (Lewis et al., 1990; Marret, 1994) y están asociados con condiciones cálidas durante el invierno y verano $\left(16\right.$ y $21^{\circ} \mathrm{C}$, respectivamente) y salinidades mayores a 36 . Sin embargo, estas especies también han sido documentadas en ambientes fríos en el Pacífico Norte (Radi et al., 2001) y Atlántico Sur (Candel et al., 2012).

Selenopemphix quanta es conocido como una especie costera (cosmopolita) de regiones polares a subtropicales (Harland, 1983; Edwards \& Andrle, 1992; Dale, 1996) (Tabla 2). A pesar que las abundancias relativas más altas se han encontrado en zonas costeras, este taxón también ocurre en áreas oceánicas abiertas. Ha sido documentado principalmente en áreas costeras del Hemisferio Norte y del Frente Subtropical del Hemisferio Sur (aproximadamente al norte de los $45^{\circ} \mathrm{S}$ ). Está distribuido dentro de un amplio rango de temperatura, salinidad y nutrientes, con valores de temperatura que varían entre $-2,1$ y $29,6^{\circ} \mathrm{C}$ (invierno-verano), salinidad entre 16,9 y 36,7 (primavera-verano) (Marret \& Zonneveld, 2003). Según Rochon et al. (1999) y de Vernal et al. (2001) esta especie muestra una mayor adaptabilidad a temperaturas que varían entre 8 y $14^{\circ} \mathrm{C}$ y salinidades entre 23 y 31.

Votadinium spinosum puede considerarse una especie templada/subtropical, característica de ambientes completamente marinos oligo a mesotróficos y presente en áreas con temperaturas restringidas entre 12,2 y $27,8^{\circ} \mathrm{C}$ (inviernoverano) y salinidades superiores a 31,7 durante todo el año. Ha sido documentado en una pequeña franja de regiones subtropicales y templadas en el Hemisferio Norte (Marret \& Zonneveld, 2003; Radi \& de Vernal, 2004, 2008; Limoges et al., 2010). Sin embargo, en el Hemisferio Sur, V. spinosum ha sido registrado en sedimentos modernos provenientes de la costa de Mar del Plata ( $38^{\circ} 28^{\prime} \mathrm{S}-57^{\circ} 41^{\prime} \mathrm{O}$; Vilanova et al., 2008) y del fondo del Canal Beagle (Candel et al., 2012).
Quistes de Pentapharsodinium dalei han sido registrados en todas las latitudes, excluyendo la región al sur de los $62^{\circ} \mathrm{S}$, desde ambientes costeros a marinos profundos (Tabla 2). Puede considerárselo una especie cosmopolita con gran participación en las asociaciones del Hemisferio Norte, mientras que es más raro en las del Hemisferio Sur. Este taxón está distribuido dentro de un amplio rango de parámetros ambientales, con valores de temperatura entre $-2,1$ y $29,6^{\circ} \mathrm{C}$ (invierno-verano) y salinidades entre 21,3 y 36,7 (primaveraverano) (Marret \& Zonneveld, 2003). Sin embargo, presenta las abundancias relativas más altas en sedimentos costeros de ambientes templados fríos a subpolares (Marret \& Zonneveld, 2003; Radi \& de Vernal, 2008).

Operculodinium centrocarpum es considerado una especie cosmopolita y se encuentra dentro de una amplia variedad de ambientes desde zonas costeras hasta oceánicas (Marret \& Zonneveld, 2003). Está distribuido dentro de un muy amplio rango de temperaturas: $-2,1$ y $29,6^{\circ} \mathrm{C}$ y salinidades: 16,1 y 36,8 (Marret \& Zonneveld, 2003). Las abundancias relativas más altas se han encontrado en el norte del Atlántico Norte (Harland, 1983; Edwards \& Andrle, 1992; Rochon et al., 1999) y regiones de la Corriente de Benguela, en la costa sur de África. Sin embargo, constituye sólo una pequeña parte de las asociaciones de dinoquistes al sur del Frente Subpolar Antártico. Similarmente, Spiniferites sp. es considerado una especie cosmopolita registrada en ambientes neríticos internos a externos, en regiones templadas a tropicales (Wall et al., 1977; Dale, 1983; Harland, 1983; Edwards \& Andrle, 1992) (Tabla 2). En Argentina, ambos taxones han sido reportados por Grill \& Guerstein (1995), Grill \& Quattrocchio (1996), Gómez et al. (2005), Borel \& Gómez (2006) y Vilanova et al. (2008) en sedimentos marinos modernos y fósiles de la Provincia de Buenos Aires $\left(36^{\circ}-38^{\circ} \mathrm{S} ; 56^{\circ}-62^{\circ} \mathrm{O}\right)$, mientras que Borromei \& Quattrocchio (2001, 2007), Grill et al. (2002) y Candel et al. $(2009,2012)$ los registraron en el sur de Tierra del Fuego.

En el presente trabajo se consideran a Polykrikos kofoidii y Polykrikos schwartzii en el sentido de Matsuoka et al. (2009), y es por ello que las condiciones ambientales previamente documentadas para estos taxa deben ser consideradas cuidadosamente. Quistes de Polykrikos schwartzii, previamente documentados como P. kofoidii (Matsuoka, 1985, 1987; Marret \& Zonneveld, 2003; Radi $\&$ de Vernal, 2004), han sido registrados en áreas costeras tropicales a subtropicales y zonas de "upwelling", a menudo, entre los $45^{\circ} \mathrm{N}$ y $45^{\circ} \mathrm{S}$. Las abundancias relativas más altas han sido encontradas en el Golfo de Guinea y en el Mar de Japón. Su distribución está restringida a áreas caracterizadas por temperaturas entre 25 y $29,1^{\circ} \mathrm{C}$ y salinidades entre 31,6 y 36,6, en áreas oligo a mesotróficas (Marret \& Zonneveld, 2003) (Tabla 2). Sin embargo, quistes de P. schwartzii (previamente citados como $P$. kofoidii) fueron documentados en los mares de Bering y Chukchi en el Pacífico Norte (Radi et al., 2001) y en los sedimentos modernos del Canal Beagle (Candel et al., 2012), sugiriendo que esta especie puede tolerar temperaturas frías. Asimismo, quistes de Polykrikos kofoidii, previamente descriptos como $P$. schwartzii, pueden 
ser considerados especies templado-frías a subtropicales, con un amplio rango de temperaturas variando entre $-0,9$ y $27,5^{\circ} \mathrm{C}$ y salinidades superiores a 28,5 distribuidas dentro de un amplio rango de ambientes, desde costeros a oceánicos abiertos y oligo a eutróficos (Marret \& Zonneveld, 2003).

\section{Ecología de los restantes palinomorfos acuáticos}

De los acritarcos, si bien se desconoce su afinidad biológica, pueden inferirse sus preferencias ecológicas a partir de su asociación con otros microfósiles. Este grupo está constituido por palinomorfos pertenecientes a los géneros Halodinium y un grupo de pequeños acritarcos de pared hialina asignables a Micrhystridium y Sigmopollis. Los registros de Halodinium provienen de depósitos marinos o estuáricos, sugiriéndose que su presencia indica una influencia fluvial fuerte y asociaciones con ambientes transicionales (de Vernal et al., 1989; Mudie, 1992; Head, 1993). Su afinidad biológica es incierta, sin embargo de Vernal et al. (1989) sugieren una afinidad con los rizópodos. El género Micrhystridium ha sido registrado frecuentemente en depósitos marinos, aunque palinomorfos de similares características (Tipo 115) han sido encontrados en sedimentos cuaternarios continentales en los Países Bajos (Pals et al., 1980). Por otro lado, Sigmopollis se asocia a condiciones de agua dulce (van Geel et al., 1983; van Der Wiel, 1982).

Dentro de las Clorofíceae, se encuentra Botryococcus con una amplia distribución en regiones tropicales y templadas, llegando a tolerar climas estacionalmente fríos (Batten \& Grenfell, 1996). Es una forma dulceacuícola y eurihalina, que prospera inclusive en albuferas (Medeanic, 2006).

Las Zygnemataceae, representadas por los géneros Spirogyra y Zygnema, son algas casi exclusivamente dulceacuícolas, raramente ocurren en ambientes salobres y tienen una distribución casi global. Son características de ambientes lénticos de aguas someras, ricas en oxígeno, mesotróficas a eutróficas con ocurrencia en lagos, lagunas, arroyos y ríos (Head, 1993; van Geel et al., 1981). Muy pocas especies habitan lagunas salobres y no existen representantes marinos (Kadlubowska, in Martínez et al., 2008).

Las Prasinofíceae (géneros Tasmanites y Cymatiosphaera) son frecuentes en ambientes marinos de salinidad reducida (Brocke \& Riegel, 1996). Prauss \& Riegel (1989) y Prauss $(1996,2001)$ sugirieron que la distribución de estas algas es favorecida no solo por aguas de salinidad reducida, sino también por una baja temperatura. Sin embargo, registros de prasinofíceas en depósitos deltaicos del Pleistoceno en latitudes bajas-medias (sub-ecuatoriales) confirman que la salinidad es el parámetro más importante que controla su distribución, restando una influencia directa a la temperatura (Brocke \& Riegel, 1996).

Respecto a los huevos de copépodos (Figura 3O), en general, han sido registrados en un amplio rango de ambientes acuáticos, desde lagos continentales a marinos, y su distribución puede estar relacionada con los efectos combinados de alta productividad y alta sedimentación (van Waveren, 1994). Son particularmente abundantes en ambientes antárticos (Borromei \& Quattrocchio, 2007). Por otro lado, los revestimientos de foraminíferos (Figura 3P) están presentes en ambientes marinos o marino-salobres de plataforma, donde la disponibilidad de alimento o nutrientes es un factor importante que controla su abundancia en los sedimentos (Tyson, 1995).

\section{DISCUSIÓN Y CONCLUSIONES}

En la Isla Grande de Tierra del Fuego el mejoramiento climático postglacial es coincidente con el ingreso del agua mar a la artesa glacial donde hoy se emplaza el Canal Beagle, el cual tuvo lugar alrededor de los 8.200 años $\mathrm{C}^{14} \mathrm{AP}$ (Rabassa et al., 2009). El ingreso del agua de mar inundó el área del Lago Roca-Bahía Lapataia (Figura 1) generándose ambientes estuarinos de agua dulce a salobre y baja energía, como resultado de la mezcla del agua marina que ingresaba paulatinamente y del agua dulce proveniente de los lagos proglaciales originados durante el retroceso de los hielos (Rabassa et al., 2000). A estos ambientes se correlacionan los niveles marinos más antiguos identificados en Bahía Lapataia (Rabassa et al., 1986) y Lago Roca (Gordillo et al., 1993) (Tabla 3), ambos sitios ubicados en el sector oeste del canal. De acuerdo con las asociaciones de palinomorfos acuáticos identificados en las localidades de Bahía Lapataia y Río Varela, ubicada unos $100 \mathrm{~km}$ al este de Bahía Lapataia (Figura 1, Tabla 3), se observa entre los ca 8.200 y 5.500 años $\mathrm{C}^{14} \mathrm{AP}$, el desarrollo de ambientes estuarinos internos a externos, con salinidades bajas y variables, temperatura del agua de mar templado-fría y abundancia de nutrientes disueltos debido al aporte de agua dulce provenientes de los glaciares de circo (Borromei \& Quattrocchio, 2001, 2007; Grill et al., 2002). Las asociaciones de palinomorfos acuáticos (Tabla 1) están caracterizadas por un bajo número de taxa y comprenden quistes de dinoflagelados de los ordenes Peridiniales (Brigantedinium spp. y Selenopemphix nephroides) y Gonyaulacales (Spiniferites sp. y Operculodinium centrocarpum), acompañados por acritarcos (Halodinium sp. y Micrhystridium sp.), revestimientos de foraminíferos, huevos de copépodos y algas dulceacuícolas (Botryococcus spp. y Pediastrum spp.). Estas últimas y la presencia de Halodinium sp., sugieren la descarga de agua dulce al medio marino, lo cual sumado a condiciones de circulación restringida, propia de ambientes de fiordos, habrían favorecido el desarrollo de salinidades sub-normales. La asociación de moluscos registrada en el área del Lago Roca-Bahía Lapataia, para este intervalo de tiempo, está caracterizada también por una escasa biodiversidad de taxa epibentónicos (Mytílidos y Cirripedios) tolerantes a condiciones estuáricas (Gordillo et al., 1993; Gordillo, 1992, 1999).

Entre los $c a 6.000$ y los 3.500 años $\mathrm{C}^{14} \mathrm{AP}$, las asociaciones de palinomorfos acuáticos reconocidos en las localidades de Albufera Lanushuaia y Río Ovando (Figura 1, Tabla 3) revelan un aumento en las especies de dinoquistes respecto de las registradas en Bahía Lapataia y Río Varela. Las asociaciones identificadas (Tabla 1) están compuestas principalmente por dinoquistes del orden Peridiniales 
(Brigantedinium simplex, B. spp., Echinidinium granulatum, E. cf. E. granulatum, E. cf. E. delicatum, E. spp., Islandinium minutum, Dubridinium cf. D. sp., Selenopemphix nephroides, S. quanta, S. cf. S. quanta, S. sp., Votadinium spinosum y Pentapharsodinium cf. $P$. dalei), acompañados por taxa Gonyaulacales (Operculodinium cf. O. centrocarpum y Spiniferites spp.) y Gymnodiniales (Polykrikos kofoidii y P. schwartzii). Además se identificaron acritarcos (Halodinium sp., Micrhystridium sp., Sigmopollis sp. y Acritarco sp.1), revestimientos de foraminíferos, huevos de copépodos y algas dulceacuícolas a marino-salobres (Spirogyra sp., Zygnema sp., Cymathiosphaeraceae y Tasmanaceae). Estas asociaciones sugieren un ambiente marino-marginal, con baja a moderada salinidad y altas concentraciones de nutrientes en las aguas marinas superficiales, probablemente debido al aporte de agua dulce por escorrentía superficial (Candel et al., 2009, 2011). Esta diversificación de especies es además sustentada por la asociación de moluscos identificada, que sugiere una importante expansión de la fauna y una mayor diversificación de las asociaciones indicando un cambio hacia las condiciones actuales (Gordillo et al., 2005).

En la localidad de Río Ovando, entre los 4.100 y 4.000 años $\mathrm{C}^{14} \mathrm{AP}$, la ocurrencia de especies de dinoquistes "oportunistas" ("bloom") pertenecientes al complejo Islandinium-Echinidinium y el incremento de algas clorofitas de agua dulce a salobre (Botryococcus sp., Spirogyra sp. y Cymatiosphaera sp.) sugieren un mayor aporte de agua dulce al canal (Candel et al., 2009; Candel, 2010). Actualmente, los géneros Islandinium y Echinidinium, que conforman dicho complejo, toleran un amplio rango de condiciones de temperatura y salinidad. Como se mencionó previamente, el género Islandinium presenta las mayores abundancias relativas en regiones con temperatura superficial del agua por debajo de $\operatorname{los} 5^{\circ} \mathrm{C}$ y una amplia tolerancia a salinidades variables, esto explica la gran abundancia en ambientes estuarinos (Head et al., 2001). Asimismo, Echinidinium es un taxón característico de ambientes con concentraciones relativamente altas de nutrientes en las aguas superficiales, como áreas de "upwelling” y regiones influenciadas por la descarga de los ríos caracterizadas por baja salinidad y estratificación en la parte superior de la columna de agua (Marret \& Zonneveld, 2003). Según Gorin \& Steffen (1991), la baja diversidad de las asociaciones de microplancton podría ser indicativo de condiciones restringidas y estrés ambiental con salinidades, a menudo, inestables. Un comportamiento similar se observa en los moluscos con el reemplazo de una asociación diversa compuesta por Venéridos y otros moluscos (bivalvos, gastrópodos y quitones), por una asociación casi monoespecífica (Mytilus y Hiatella) tolerantes a salinidades bajas o variables. Estas evidencias paleoecológicas indicarían ambientes marinos muy restringidos y con un alto aporte estacional de agua dulce por descarga de los ríos y/o por derretimiento glacial (Candel et al., 2009). De acuerdo con las asociaciones de palinomorfos acuáticos actuales observadas en las muestras de fondo de canal, esta asociación de dinoquistes "oportunistas" no tendrían análogos modernos en el Canal Beagle (Candel et al., 2012).
En síntesis, luego del retroceso de los hielos, el nivel relativo del mar comenzó a ascender e inundar paulatinamente el Canal Beagle, a los 8.200 años AP, convirtiendo el canal en un profundo y angosto fiordo. La incursión marina alcanzó su punto máximo entre los 5.000-6.000 años $\mathrm{C}^{14} \mathrm{AP}$ (Gordillo et al., 1993). El posterior proceso regresivo no fue linear, dejando varias terrazas elevadas discontinuas. La causa de estos cambios es atribuida a efectos tectónicos diferenciales y movimientos corticales glacioisostáticos. El análisis de los palinomorfos acuáticos identificados en las distintas localidades (Bahía Lapataia y Río Ovando en el sector oeste; Albufera Lanushuaia y Río Varela en el sector este) muestran que los ambientes desarrollados durante el inicio de la ingresión marina del Holoceno en el Canal Beagle, correspondieron a ambientes estuarinos con salinidades anormalmente bajas y variables caracterizados, en particular, por asociaciones de dinoquistes poco diversas. A medida que se instaló el ambiente marino, entre los 5.800 y 3.500 años $\mathrm{C}^{14} \mathrm{AP}$, la asociación de microplancton fue incrementando el número de especies, principalmente taxa de dinoquistes Peridiniales, sugiriendo el establecimiento de ambientes marino-marginales con salinidades bajas a moderadas y alto contenido de nutrientes en las aguas. Las asociaciones de mayor diversidad registradas a partir de los 6.000 años $\mathrm{C}^{14} \mathrm{AP}$, muestran una composición similar con las asociaciones de palinomorfos acuáticos actuales reconocidas en los sedimentos modernos del Canal Beagle, dominadas principalmente por Brigantedinium spp., Echinidinium spp. y Selenopemphix quanta, acompañadas por Pentapharsodinium dalei, Islandinium minutum, Votadinium spinosum, Polykrikos kofoidii y Polykrikos schwartzii (Candel et al., 2012). Asimismo, estas asociaciones de microplancton son comparables con las asociaciones modernas características de las plataformas continentales del Océano Ártico, fuertemente influenciada por los aportes de agua dulce de los ríos principalmente durante el verano.

\section{AGRADECIMIENTOS}

Los autores desean agradecer a Jorge Rabassa y Andrea Coronato (CADIC-CONICET), por la ayuda brindada durante las tareas de campo. A M. Quattrocchio por la lectura del manuscrito, sus comentarios y sugerencias. A C.C. Lana por su amable ayuda en la revisión del resumen en portugués. Este trabajo fue financiado por los proyectos PICTR 2002-00067 (ANPCyT) y PIP-CONICET 02787/02.

\section{REFERENCIAS}

Albero, M.C.; Angiolini, F. \& Piana, L. 1988. Holocene ${ }^{14} \mathrm{C}$ reservoir effect at Beagle Channel (Tierra del Fuego, Argentina Republic). Quaternary of South America and Antarctic Peninsula, 5:59-71.

Batten, D.J. \& Grenfell, H.R. 1996. Capítulo 7D. Botryococcus. In: J. Jansonius \& D.C. McGregor (eds.) Palynology: principles and applications, American Association of Stratigraphic Palynologists Foundation, 1:205-214.

Borel, C.M. 2007. Algas no silíceas y acritarcos de depósitos costeros holocenos en el arroyo La Ballenera, Buenos Aires, Argentina. Ameghiniana, 44:359-366. 
Borel, C.M.; Boltovskoy, A.; Guerstein, G.R. \& Stutz, S. 2006. Quistes de dinoflagelados no marinos del Holoceno de la provincia de Buenos Aires, Argentina: relación con especies actuales de Peridinium. Revista del Museo Argentino de Ciencias Naturales, 8:119-125.

Borel, C.M. \& Gómez, E.A. 2006. Palinología del Holoceno del Canal del Medio, estuario de Bahía Blanca (Buenos Aires, Argentina). Ameghiniana, 43:399-412.

Borel, C.M.; Guerstein, G.R. \& Prieto, A.R. 2003. Palinomorfos acuáticos (algas y acritarcos) del Holoceno de la laguna Hinojales (Buenos Aires, Argentina): interpretación paleoecológica. Ameghiniana, 40:531-544.

Borromei, A.M. \& Quattrocchio, M. 2001. Palynological study of Holocene marine sediments from Bahía Lapataia, Beagle Channel, Tierra del Fuego, Argentina. Revista Española de Micropaleontología, 33:61-70.

Borromei, A.M. \& Quattrocchio, M. 2007. Holocene sea-level change and marine palynology of the Beagle Channel, southern Tierra del Fuego, Argentina. Ameghiniana, 44:161-171.

Brocke, R. \& Riegel, W. 1996. Phytoplankton responses to shoreline fluctuations in the Upper Muschelkak (Middle Triassic) of Lower Saxony (Germany). Neues Jahrbuch für Geologie und Paläontologie, 200:53-73.

Bujalesky, G.; Coronato, A.; Roig, C. \& Rabassa, J. 2004. Holocene differential tectonic movements along the Argentine sector of the Beagle Channel (Tierra del Fuego) inferred from marine palaeoenvironments. Bollettino de Geofisica Teorica ed Applicata, 45:235-238.

Candel, M.S. 2010. Cambios relativos del nivel del mar en el Canal Beagle, Tierra del Fuego (Cenozoico Tardio), en base al análisis palinológico. Universidad Nacional del Sur, Tesis Doctoral, 194 p.

Candel, M.S.; Borromei, A.M.; Martínez, M.A.; Gordillo, S.; Quattrocchio, M. \& Rabassa, J. 2009. Middle-Late Holocene palynology and marine mollusks from Archipiélago Cormoranes area, Beagle Channel, southern Tierra del Fuego, Argentina. Palaeogeography, Palaeoclimatology, Palaeoecology, 273:111122. doi:10.1016/j.palaeo.2008.12.009.

Candel, M.S.; Borromei, A.M.; Martínez, M.A. \& Bujalesky, G. 2013. Palynofacies analysis of surface sediments from Beagle Channel and its application as modern analogues for Holocene records of Tierra del Fuego, Argentina. Palynology, 37:62-76. doi:10.1080/01916122.2012.718994.

Candel, M.S.; Martínez, M.A. \& Borromei, A.M. 2011. Palinología y palinofacies de una secuencia marina del Holoceno mediotardío: Albufera Lanushuaia, Canal Beagle, Tierra del Fuego, Argentina. Revista Brasileira de Paleontología, 14:297-310. doi:10.4072/rbp.2011.3.09.

Candel, M.S.; Radi, T.; de Vernal, A. \& Bujalesky, G. 2012. Distribution of dinoflagellate cysts and other aquatic palynomorphs in surface sediments from the Beagle Channel, southern Argentina. Marine Micropaleontology, 96-97:1-12. doi:10.1016/j.marmicro.2012.06.009.

Coronato, A.; Rabassa, J.; Borromei, A.; Quattrocchio, M. \& Bujalesky, G. 1999. Nuevos datos sobre el nivel relativo del mar durante el Holoceno en el Canal Beagle, Tierra del Fuego, Argentina. In: CONGRESO ARGENTINO DE CUATERNARIO Y GEOMORFOLOGÍA, 1, 1999. Actas, 1:27-28.

Dale, B. 1976. Cyst formation, sedimentation, and preservation: factors affecting dinoflagellate assemblages in Recent sediments from Trondheimsfjord, Norway. Review of Palaeobotany and Palynology, 22:39-60. doi:10.1016/0034-6667(76)90010-5.
Dale, B. 1983. Dinoflagellate resting cysts: "benthic plankton". In: G.A. Fryxell (ed.) Survival Strategies of the Algae, Cambridge University Press, p. 69-135.

Dale, B. 1996. Dinoflagellate cysts ecology: modeling and geological applications. In: J. Jansonius \& D.C. McGregor (eds.) Palynology: Principles and Applications, American Association of Stratigraphic Palynologists Foundation, UT, 3:1249-1275.

de Vernal, A.; Eynaud, F.; Henry, M.; Hillaire-Marcel, C.; Londeix, L.; Mangin, S.; Matthiessen, J.; Marret, F.; Radi, T.; Rochon, A.; Solignac, S. \& Turon, J.-L. 2005. Reconstruction of seasurface conditions at middle to high latitudes of the Northern Hemisphere during the Last Glacial Maximum (LGM) based on dinoflagellate cyst assemblages. Quaternary Science Reviews, 24:897-924. doi:10.1016/j.quascirev.2004.06.014.

de Vernal, A.; Goyette, C. \& Rodrigues, C.G. 1989. Contribution palynostratigraphique (dinokystes, pollen et spores) à la connaissance de la Mer Champlain: coupe de Saint-Césaire, Quebec. Canadian Journal of Earth Sciences, 26:2450-2464.

de Vernal, A.; Henry, M.; Matthiessen, J.; Mudie, P.; Rochon, A.; Boessenkool, K.; Eynaud, F.; Grøsfjeld, K.; Guiot, J.; Hamel, F.; Harland, R.; Head, M.; Kunz-Pirrung, M.; Levac, E.; Loucheur, V.; Peyron, O.; Pospelova, V.; Radi, T; Turon, J-L. \& Voronina, E. 2001. Dinoflagellate cyst assemblages as tracers of sea-surface conditions in the northern North Atlantic, Arctic and sub-Arctic seas: the new ' $n=677$ ' data base and its application for quantitative palaeoceanographic reconstruction. Journal of Quaternary Science, 16:681-698. doi:10.1002/jqs.659.

de Vernal, A. \& Mudie, P.J. 1989. Late Pliocene to Holocene Palynostratigraphy at ODP site 645, Baffin Bay. Proceeding of the Ocean Drilling Program, Scientific Results, 105:387-399.

Edwards, L. \& Andrle, A.S. 1992. Distribution of selected dinoflagellate cysts in modern marine sediments. In: M.J. Head \& L.H. Wrenn (eds.) Neogene and Quaternary Dinoflagellate Cysts and Acritarchs, American Association of Stratigraphic Palynologists Foundation, 259-288 p.

Fensome, R.A. \& Williams, G.L. 2004. The Lentin and Williams index of fossil dinoflagellates 2004 edition. Pittsburgh, American Association of Stratigraph Palynologists, 909 p. (Contribution Series 42).

Gómez, E.A.; Martínez, D.E.; Borel, C.M.; Guerstein, G.R. \& Cusminsky, G.C. 2005. Submarine evidence of Holocene sea level fluctuations in the Bahía Blanca estuary, Argentina. Journal of South American Earth Sciences, 20:139-155. doi:10.1016/ j.jsames.2005.06.011.

Gordillo, S. 1992. Tafonomía y paleoecología de moluscos bivalvos del Holoceno del Canal Beagle, Tierra del Fuego. Universidad Nacional de Córdoba, Tesis Doctoral, 286 p.

Gordillo, S. 1993. Las terrazas marinas holocenas de la región del Beagle (Tierra del Fuego) y su fauna asociada. In: CONGRESO GEOLÓGICO ARGENTINO, 12, 1993. Actas, 6:34-39.

Gordillo, S. 1999. Holocene molluscan assemblages in the Magellan region. Scientia Marina, 63:15-22.

Gordillo, S.; Bujalesky, G.; Pirazzoli, P.; Rabassa, J. \& Saliége, J. 1992. Holocene raised beaches along the northern coast of the Beagle Channel, Tierra del Fuego, Argentina. Palaeogeography, Palaeoclimatology, Palaeoecology, 99:41-54. doi:10.1016/00310182(92)90006-Q.

Gordillo, S.; Coronato, A. \& Rabassa, J. 1993. Late Quaternary evolution of a subantarctic paleofjord, Tierra del Fuego. Quaternary Science Reviews, 12:889-897. doi:10.1016/02773791(93)90027-J. 
Gordillo, S.; Coronato, A. \& Rabassa, J. 2005. Quaternary molluscan faunas from the island of Tierra del Fuego after the Last Glacial Maximum. Scientia Marina, 69:337-348.

Gorin, G.E. \& Steffen D. 1991. Organic facies as a tool for recording eustatic variations in marine fine-grained carbonates - example of the Berriasian stratotype at Berrias (Ardèche, SE France). Palaeogeography, Palaeoclimatology, Palaeoecology, 85:303320. doi:10.1016/0031-0182(91)90164-M.

Grill, S.; Borromei, A.M.; Quattrocchio, M.; Coronato, A.; Bujalesky, G. \& Rabassa, J. 2002. Palynological and sedimentological analysis of Recent sediments from Río Varela, Beagle Channel, Tierra del Fuego, Argentina. Revista Española de Micropaleontología, 34:145-161.

Grill, S. \& Guerstein, G.R. 1995. Estudio palinológico de sedimentos superficiales en el estuario de Bahía Blanca, provincia de Buenos Aires, Argentina. Polen, 7:41-49.

Grill, S. \& Quattrocchio, M., 1996. Fluctuaciones eustáticas durante el Holoceno a partir del análisis de palinomorfos. Localidad: Grümbein. Sur de la provincia de Buenos Aires. Ameghiniana, 33:435-442.

Guy-Ohlson, D. 1996. Capítulo 7B. Prasinophycean algae. In: J. Jansonius \& D.C. McGregor (eds.) Palynology: principles and applications. American Association of Stratigraphic Palynologists Foundation, 1:181-189.

Guy-Ohlson, D. \& Boalch, G.T. 1992. Comparative morphology of the genus Tasmanites (Pterospermales, Chlorophyta). Phycologia, 31:523-528. doi:10.2216/i0031-8884-31-6-523.1.

Harland, R. 1983. Distribution maps of recent dinoflagellate cysts in bottom sediments from the North Atlantic Ocean and adjacent seas. Palaeontology, 26:321-387.

Harland, R. \& Pudsey, C.J. 1999. Dinoflagellate cysts from sediment traps deployed in the Bellinghausen, Weddell and Scotia seas, Antarctica. Marine Micropaleontology, 37:77-99. doi.org/10.1016/S0377-8398(99)00016-X.

Harland, R.; Pudsey, C.J.; Howe, J.A. \& Fitzpatrick, M.E.J. 1998. Recent dinoflagellate cysts in a transect from the Falkland Trough to the Weddell Sea, Antarctica. Palaeontology, 41:1093-1131.

Head, M.J. 1993. Dinoflagellates, Sporomorphs, and Other Palynomorphs from the Upper Pliocene St. Erth Beds of Cornwall, Southwestern England. Journal of Paleontology, 67:1-62.

Head, M.; Harland, R. \& Matthiessen, J. 2001. Cold marine indicators of the late Quaternary: the new dinoflagellate cyst genus Islandinium and related morphotypes. Journal of Quaternary Science, 16:621-636. doi:10.1002/jqs.657.

Heusser, L. \& Stock, C. 1984. Preparation techniques for concentrating pollen from marine sediments and other sediments with low pollen density. Palynology, 8:225-227. doi:10.1080/0 1916122.1984.9989279.

Lewis, J.; Dodge, J.D. \& Powell, A.J. 1990. Quaternary dinoflagellate cysts from the upwelling system offshore Peru, hole 686B, ODP Leg 112. Proceeding of the Ocean Drilling Program, Scientific Results, 112:323-327.

Limoges, A.; Kielt, J.-F.; Radi, T.; Ruíz-Fernandez, A.C. \& de Vernal, A. 2010. Dinoflagellate cyst distribution in surface sediments along the south-western Mexican coast $\left(14.76^{\circ} \mathrm{N}\right.$ to $\left.24.75^{\circ} \mathrm{N}\right)$. Marine Micropaleontology, 76:104-123. doi:10.1016/j. marmicro.2010.06.003.

Marret, F. 1994. Distribution of dinoflagellate cysts in recent marine sediments from the east Equatorial Atlantic (Gulf of Guinea). Review of Palaeobotany and Palynology, 84:1-22. doi:org/10.1016/0034-6667(94)90038-8.
Marret, F. \& de Vernal, A., 1997. Dinoflagellate cyst distribution in surface sediments of the southern Indian Ocean. Marine Micropaleontology, 29:367-392. doi:org/10.1016/S03778398(96)00049-7.

Marret, F.; de Vernal, A.; Benderra, F. \& Harland, R. 2001. Late Quaternary sea-surface conditions at DSDP Hole 594 in the southwest Pacific Ocean based on dinoflagellate cyst assemblages. Journal of Quaternary Science, 16:739-751. doi:10.1002/jqs.648.

Marret, F. \& Zonneveld, K.A.F., 2003. Atlas of modern organic-walled dinoflagellate cyst distribution. Review of Palaeobotany and Palynology, 125:1-200. doi.org/10.1016/ S0034-6667(02)00229-4.

Martínez, M.A.; Ferrer, N.C. \& Asensio, M.A. 2008. Primer registro de algas dulceacuícolas del Paleógeno de la Cuenca de Nirihuau, Argentina: descripciones sistemáticas y análisis palinofacial. Ameghiniana, 45:719-735.

Matsuoka, K. 1985. Archeopyle structure in modern Gymnodinialean dinoflagellate cysts. Review of Palaeobotany and Palynology, 44:217-231. doi:org/10.1016/0034-6667(85)90017-X.

Matsuoka, K., 1987. Organic-walled dinoflagellate cysts from surface sediments of Akkeshi Bay and Lake Saroma, North Japan. Bulletin of the Faculty of Liberal Arts, Nagasaki University, Natural Science, 28:35-123.

Matsuoka, K.; Kawami, H.; Nagai, S.; Iwataki, M. \& Takayama, H. 2009. Re-examination of cyst-motile relationships of Polykrikos kofoidii Chatton and Polykrikos schwartzii Bütschli (Gymnodiniales, Dinophyceae). Review of Palaeobotany and Palynology, 154:79-90. doi:org/10.1016/j. revpalbo.2008.12.013.

Matthiessen, J. 1995. Distribution patterns of dinoflagellate cysts and other organic-walled microfossils in recent NorwegianGreenland Sea sediments. Marine Micropaleontology, 24:307334. doi:org/10.1016/0377-8398(94)00016-G.

McMinn, A. 1992. Pliocene through Holocene dinoflagellate cyst biostratigraphy of the Gippsland Basin, Australia. In: M. Head \& J. Wrenn (eds) Neogene and Quaternary Dinoflagellate and Acritarchs, American Association of Stratigraphic Palynologist Foundation, p. 141-161.

McMinn, A. 1995. Why are there no post-Palaeogene dinoflagellate cysts in the Southern Ocean? Micropaleontology, 41:383-386.

McMinn, A. \& Wells, P. 1997. Use of dinoflagellate cysts to determine changing Quaternary sea-surface temperature in southern Australia. Marine Micropaleontology, 29:407-422. doi:org/10.1016/S0377-8398(96)00012-6.

Medeanic, S. 2006. Freshwater algal palynomorph records from Holocene deposits in the coastal plain of Rio Grande do Sul, Brazil. Review of Palaeobotany and Palynology, 141:83-101. doi:org/10.1016/j.revpalbo.2006.03.012.

Mudie, P.J. 1992. Circum-arctic Quaternary and Neogene marine palynofloras: paleoecology and statistical analysis. In: M.J. Head \& J.H. Wrenn (eds.) Neogene and Quaternary Dinoflagellate Cysts and Acritarchs, American Association Stratigraphic Palynologists Foundation, p. 347-390

Pals, J.P.; van Geel, B. \& Delfos, A. 1980. Paleoecological studies in the Klokkeweel bog near Hoogkarspel (Prov. of NoordHolland). Review of Palaeobotany and Palynology, 30:371-418. doi:org/10.1016/0034-6667(80)90020-2.

Prauss, M. 1996. The Lower Toarcian Posidonia Shale of Grimmen, Northeast Germany. Implications from the palynological analysis of a near-shore section. Neues Jahrbuch für Geologie und Paläontologie, Abhandlungen, 200:107-132. 
Prauss, M. 2001. Sea-Level changes and organic-walled phytoplankton response in a Late Albian epicontinental setting, Lower Saxony basin, NW Germany. Palaeogeography, Palaeoclimatology, Palaeoecology, 174:221-249. doi:org/10.1016/S00310182(01)00295-4.

Prauss, M. \& Riegel, W. 1989. Evidence from phytoplankton associations for causes of black shale formation in epicontinental seas. Neues Jahrbuch für Geologie und Paläontologie, Mh, 11:671-682.

Rabassa, J.; Bujalesky, G.; Meglioli, A.; Coronato, A.; Gordillo, S.; Roig, C. \& Salemme, M. 1992. The Quaternary of Tierra del Fuego, Argentina: the status of our knowledge. Sveriges Geologiska Undersköning, 81:249-256.

Rabassa, J.; Coronato, A.; Bujalesky, G.; Salemme, M.; Roig, C.; Meglioli, A.; Heusser, J.; Gordillo, S.; Roig, F.; Borromei, A. \& Quattrocchio, M. 2000. Quaternary of Tierra del Fuego, Southernmost South America: an updated review. Quaternary International, 68-71:217-240. doi:org/10.1016/S10406182(00)00046-X.

Rabassa, J.; Coronato, A.; Gordillo, S.; Candel, M.S. \& Martínez, M.A. 2009. Paleoambientes litorales durante el inicio de la transgresión marina holocena en Bahía Lapataia, Canal Beagle, Parque Nacional Tierra del Fuego. Revista de la Asociación Geológica Argentina, 65:648-659.

Rabassa, J.; Coronato, A. \& Martínez, O. 2011. Late Cenozoic glaciations in Patagonia and Tierra del Fuego: an updated review. Biological Journal of the Linnean Society, 103:316-335.

Rabassa, J.; Coronato, A.; Roig, C.; Martínez, O. \& Serrat, D. 2004. Un bosque sumergido en Bahía Sloggett, Tierra del Fuego, Argentina: evidencias de comportamiento tectónico diferencial en el Holoceno tardío. Procesos geomorfológicos y evolución costera. In: REUNIÓN DE GEOMORFOLOGÍA LITORAL, 2, 2004. Actas, Universidad de Santiago de Compostela, p. 333-346.

Rabassa, J.O.; Heusser, C.J. \& Stuckenrath, R. 1986. New data on Holocene sea transgression in the Beagle Channel: Tierra del Fuego, Argentina. Quaternary of South America and Antarctic Peninsula, 4:291-309.

Rabassa, J.; Serrat, D.; Martí, C. \& Coronato, A. 1990. El Tardiglacial en el Canal Beagle, Tierra del Fuego, Argentina. In: CONGRESO GEOLÓGICO ARGENTINO, 11, 1990. Actas, Tierra del Fuego, 1:290-293.

Radi, T. \& de Vernal, A. 2004. Dinocyst distribution in surface sediments from the northeastern Pacific margin $\left(40-60^{\circ} \mathrm{N}\right)$ in relation to hydrographic conditions, productivity and upwelling. Review of Palaeobotany and Palynology, 128:169-193. doi:10.1016/S0034-6667(03)00118-0.

Radi, T. \& de Vernal, A. 2008. Cysts as proxy of primary productivity in mid-high latitudes of the Northern Hemisphere. Marine Micropaleontology, 68:84-114. doi: 10.1016/j. marmicro.2008.01.012.

Radi, T.; de Vernal, A. \& Peyron, O. 2001. Relationships between dinocyst assemblages in surface sediments and hydrographic conditions in the Bering and Chukchi seas. Journal of Quaternary Science, 16:667-680. doi:10.1002/jqs.652.

Radi, T.; Pospelova, V.; de Vernal, A. \& Barrie, J.V. 2007. Dinoflagellate cysts as indicators of water quality and productivity in British Columbia estuarine environments. Marine Micropaleontology, 62:269-297. doi:org/10.1016/j. marmicro.2006.09.002.
Rochon, A.; de Vernal, A.; Turon, J.L.; Matthiessen, J. \& Head, M.J. 1999. Distribution of dinoflagellate cysts in surface sediments from the North Atlantic Ocean and adjacent seas in relation to sea-surface parameters. American Association of Stratigraph Palynologists, Contribution Series, 35:152 p.

Rossignol, M. 1964. Hystrichosphères du Quaternaire en Méditerranée orientale, dans les sédiments pléistocènes et les boues marines actuelles. Revue de Micropaléontologie, 7:83-99.

Sarjeant, W.A.S. 1970. The Genus Spiniferites Mantell, 1850 (Dinophyceae). Grana, 10:74-78.doi:10.1080/00173137009429857.

Tyson, R. 1995. Sedimentary organic matter. Londres, Chapman \& Hall Eds., 615 p.

van der Wiel, A.M. 1982. Sea-Level Change and Water-Level Movements in The Netherlands During the Holocene. Vrije Universiteit, Ph.D. thesis, 138 p.

van Geel, B.; Bohncke, S.J.P. \& Dee, H. 1981. A palaeoecological study of an upper Late Glacial and Holocene sequence from "De Borchert", The Netherlands. Review of Palaeobotany and Palynology, 31:367-448. doi:org/10.1016/0034-6667(80)90035-4.

van Geel, B.; Hallewas, D.P. \& Pals, J.P. 1983. A late Holocene deposit under the Westfriese Zeedijk near Enkhuizen (Prov. of Noord-Holland, The Netherlands): palaeoecological and archaeological aspects. Review of Palaeobotany and Palynology, 38:269-335. doi:org/10.1016/0034-6667(83)90026-X.

van Geel, B. \& van der Hammen, T. 1978. Zygnemataceae in Quaternary Colombian sediments. Review of Palaeobotany and Palynology, 25:377-392. doi:org/10.1016/0034-6667(78)90021-0.

van Waveren, I.M. 1994. Distribution of copepod egg-envelopes in sub- Recent sediments from the Banda Sea (Indonesia). Scripta Geologica, 105:53-67.

Vilanova, I.; Guerstein, G.R.; Akselman, R. \& Prieto, A.R. 2008. Mid- to Late Holocene organic-walled dinoflagellate cysts from the northern Argentine shelf. Review of Palaeobotany and Palynology, 152:11-20. doi:10.1016/j.revpalbo.2008.03.006.

Vink, A.; Zonneveld, A.F. \& Willems, H. 2000. Organic-walled dinoflagellate cysts in western equatorial Atlantic surface sediments: distributions and their relation to environment. Review of Palaeobotany and Palynology, 112:247-286. doi:org/10.1016/S0034-6667(00)00046-4.

Wall, D.; Dale, B.; Lohman, G.P. \& Smith, W.K. 1977. The environmental and climatic distribution of dinoflagellate cysts in the North and South Atlantic Oceans and adjacent seas. Marine Micropaleontology, 2:121-200. doi:org/10.1016/0377-8398(77)90008-1.

Zamaloa, M. del C. 1996. Asociación de zigósporas de Zygnemataceae (Chlorophyta) en el Terciario Medio de Tierra del Fuego, Argentina. Ameghiniana, 33:179-184.

Zonneveld, K.A.F. 1997. New species of organic walled dinoflagellate cysts from modern sediments of the Arabian Sea (Indian Ocean). Review of Palaeobotany and Palynology, 97:319-337. doi:org/10.1016/S0034-6667(97)00002-X.

Zonneveld, K.A.F.; Hoek, R.; Brinkhuis, H. \& Willems, H. 2001. Geographical distributions of organic-walled dinoflagellate cysts in surficial sediments of the Benguela upwelling region and their relationship to upper ocean conditions. Progress in Oceanography, 48:25-72. doi:org/10.1016/S00796611(00)00047-1.

Received in October, 2012; accepted in July, 2013. 